\title{
Radiation-pressure Waves and Multiphase Quasar Outflows
}

\author{
Lluís Mas-Ribas ${ }^{1,2,3}$ (iD \\ ${ }^{1}$ Jet Propulsion Laboratory, California Institute of Technology, 4800 Oak Grove Dr, Pasadena, CA 91109, USA; 1luis.mas-ribas@jpl.nasa.gov \\ ${ }^{2}$ California Institute of Technology, 1200 E. California Blvd, Pasadena, CA 91125, USA \\ ${ }^{3}$ Institute of Theoretical Astrophysics, University of Oslo, Postboks 1029, NO-0315 Oslo, Norway \\ Received 2019 January 18; revised 2019 August 25; accepted 2019 August 30; published 2019 November 1
}

\begin{abstract}
We report on quasar outflow properties revealed by analyzing more than 60 composite outflow spectra built from $\sim 60,000$ C IV absorption troughs in the SDSS-III/BOSS DR12QBAL catalog. We assess the dependences of the equivalent widths of many outflow metal absorption features on outflow velocity, trough width and position, and quasar magnitude and redshift. The evolution of the equivalent widths of the $\mathrm{O}$ VI and $\mathrm{N} V$ lines with outflow velocity correlates with that of the mean absorption-line width, the outflow electron density, and the strength of lines arising from collisionally excited metastable states. None of these correlations are found for the other high- or low-ionization species, and different behaviors with trough width are also suggested. We find no dependence on quasar magnitude or redshift in any case. All the observed trends can be reconciled by considering a multiphase stratified outflow structure, where inner regions are colder, denser, and host lower-ionization species. Given the prevalence of radiative acceleration in quasar outflows found by Mas-Ribas \& Mauland, we suggest that radiation pressure sweeps up and compresses the outflowing gas outward, creating waves or filaments where the multiphase stratified structure could take form. This scenario is supported by the suggested correlation between electron density and outflow velocity, as well as by the similar behavior observed for the line and line-locking components of the absorption features. We show that this outflow structure is also consistent with other X-ray, radiative transfer, and polarization results, and discuss the implications of our findings for future observational and numerical quasar outflow studies.
\end{abstract}

Key words: galaxies: nuclei - ISM: jets and outflows - ISM: structure - quasars: absorption lines - quasars: supermassive black holes - waves

\section{Introduction}

The spectra of a fraction of about $20 \%-30 \%$ of quasars show broad absorption lines (BALs) of high-ionization species on the blue side of the corresponding quasar emission lines (Hewett \& Foltz 2003; Reichard et al. 2003; Ganguly \& Brotherton 2008; Knigge et al. 2008; Allen et al. 2011). Because these broad absorption troughs can partially mask the quasar emission lines, they are assumed to trace outflows outside the quasar broad-line region (BLR), where the emission lines are formed. Specifically, these outflows are thought to consist of the material that was orbiting the central supermassive black hole and has been accelerated outward (e.g., Murray et al. 1995; Elvis 2000; Proga et al. 2000; Proga \& Kallman 2004).

BALs appear blueshifted from the emission sources, by velocities of up to $10 \%-20 \%$ of the speed of light (Weymann et al. 1981; Trump et al. 2006; Gibson et al. 2009; Rodríguez Hidalgo et al. 2011), and about $20 \%$ of them are detached from the corresponding emission lines (Korista et al. 1993). In most cases, only broad features of high-ionization species (HiBALs) are observed (Weymann et al. 1991; Filiz et al. 2013), but a fraction of about $15 \%$ of outflows (Sprayberry \& Foltz 1992) also show BALs of low ions (LoBALs; Voit et al. 1993; Gibson et al. 2009).

Studies of BALs indicate that quasar outflows generally consist of large column densities of highly ionized material, with $\log \left(N_{\mathrm{H}} / \mathrm{cm}^{-2}\right) \gtrsim 22-23$ (e.g., Moravec et al. 2017), and supersolar metallicities (Hamann 1998). Because the absorption lines are often saturated but not completely black, it is believed that the outflows partially cover the emission sources, the exact value depending on the ionic species and line of sight (Hamann et al. 1993; Arav et al. 1999). For such a behavior in disk winds, see Proga et al. (2012). However, the bottom of the absorption troughs could also be (additionally) filled with radiation from other directions that has been scattered by the outflow into the line of sight (Lee \& Blandford 1997; Baek et al. 2007). Although it is clear that these outflows likely reside outside the BLR, their extent is still uncertain by more than two orders of magnitude, ranging from $\lesssim 1 \mathrm{pc}$ to hundreds of pc (Arav et al. 2018; Hamann et al. 2019; Proga et al. 2012).

Quasar outflows have typically been detected and studied through the analysis of BALs at ultraviolet (UV) frequencies in the quasar rest frame (Lynds 1967). Nowadays, however, the outflow absorption signatures show a wide range of complex profile shapes and widths, and they are detected in both UV and $\mathrm{X}$-ray. Specifically, BALs refer to absorption troughs blueshifted by more than $3000 \mathrm{~km} \mathrm{~s}^{-1}$ from the respective quasar emission line, and with a full width at half minimum larger than $\sim 2000 \mathrm{~km} \mathrm{~s}^{-1}$ (Weymann et al. 1991). When the features are narrower, down to a few hundreds of $\mathrm{km} \mathrm{s}^{-1}$, but one can still "decide" that they correspond to quasar outflows, these are called mini-BALs. Below that poorly defined threshold, the troughs are named narrow absorption lines (NALs; e.g., Hamann \& Sabra 2004). This classification arises from differences in the properties measured for these absorbers, but it is possible that they all trace the same type of outflows and the differences are driven by orientation, time of observation, or spatial location within the outflow; see Hamann et al. (2019) and references therein. We will refer to all the absorbers in this work as troughs, and we will analyze them independently of the aforementioned nomenclature. We will show that the distinct type of absorbers do indeed trace different media within the outflow, and that they arise naturally 
when considering the evolution of the equivalent widths of hydrogen and metal lines with outflow parameters.

In this paper, we aim to gain insight into the physical properties of quasar outflows represented by absorption troughs via the analysis of composite outflow spectra. By doing so, we will neither reveal properties of the individual objects, which are known to vary broadly, nor their time dependence, which can be of an order ranging from days to years or more (e.g., Proga et al. 2012; He et al. 2017; Misawa et al. 2018; Rogerson et al. 2018). Instead, we will obtain the time-averaged mean properties of the outflow population and will be able to analyze the dependences of these properties on several parameters in a general fashion. Furthermore, the stacking of many spectra will result in the reduction of the average spectral noise and will enable us to detect faint absorption features associated with the outflows. This is especially important in the spectral region of the $\operatorname{Ly} \alpha$ forest, where the high density of intervening absorption lines overlaps with the outflow features, making the detection of the latter difficult even in high-resolution spectra. In particular, current analyses that make use of the Ly $\alpha$ forest often have to discard the whole spectrum of quasars containing BALs ( $10 \%$ of the spectra), as the outflow contamination in this region is unknown-e.g., the cross-correlation studies by Busca et al. (2013), Font-Ribera et al. (2013), Bautista et al. (2017), and Pérez-Ràfols et al. (2018a, 2018b), or future work by DESI Collaboration et al. (2016). In these cases, our composite spectra can be used as absorption templates to mask the regions affected by the outflow, thus enabling the use of the uncontaminated spectral regions.

A number of works have analyzed composite outflow spectra (e.g., Baskin et al. 2013, 2015; Hamann et al. 2019) but they have mostly focused on correlations between the outflows and the emission sources. We present here a complementary approach: our analysis focuses on correlations and dependences between physical parameters describing the absorption troughs, such as their position in velocity space (which we assume to trace the velocity of the outflow), their width, and the degree of detachment between troughs and sources, as well as the dependences on quasar redshift and brightness.

In this work, we build upon our previous paper, Mas-Ribas \& Mauland (2019), where we constructed a number of the stacks that we will analyze here, and where we studied the observational signature of radiative acceleration known as line locking (Milne 1926; Scargle 1973; Arav \& Li 1994). In that work, we found that the mean absorption lines in the outflow composite spectra present an absorption feature on their blue side, which we identified as the C IV line-locking component. Line locking was visible in all our stacked spectra, suggesting that radiation pressure is a prevalent mechanism for the acceleration of outflows. However, we found no evidence for the presence of line locking from other species, such as O VI, $\mathrm{NV}$, or Si IV. We will consider the two components of each absorption transition (line and line locking) separately in our current analysis, and will investigate whether the properties of the outflows reveal the cause of our findings in the previous paper.

In Section 2, we present the composite spectra used for our analysis, and we detail the methods for their computation and analysis in Section 3. The overall composite outflow spectra are presented in Section 4, and we analyze the dependences of the equivalent widths on quasar and outflow parameters in Section 5 . We propose a general structure for the outflow in order to explain our measurements in Section 6. We discuss our findings and future work in Section 7, before concluding in Section 8.

We assume a flat $\Lambda \mathrm{CDM}$ cosmology with the parameter values from Planck Collaboration et al. (2016).

\section{Quasar Outflow Data}

We use the composite outflow absorption spectra of MasRibas \& Mauland (2019), built from $\approx 60,000$ broad $\left(>450 \mathrm{~km} \mathrm{~s}^{-1}\right) \mathrm{C}$ IV absorption troughs identified in the quasar spectra of the twelfth data release of the SDSS-III/BOSS (Eisenstein et al. 2011; Dawson et al. 2013) quasar catalog (DR12Q; Pâris et al. 2017). We refer to the catalog of C IV troughs as the BAL catalog, for consistency with the nomenclature in Pâris et al. (2017), DR12QBAL, although not all the troughs are BALs if strictly considering the definitions by, e.g., Weymann et al. (1991), Hall et al. (2002), and Trump et al. (2006). The DR12QBAL catalog quotes the parameters of the absorption troughs when these have widths of $>450 \mathrm{~km} \mathrm{~s}^{-1}$ with normalized quasar flux (i.e., the transmission defined by Pâris et al. 2012) below 0.9 (Pâris et al. 2017).

In detail, we analyze the 36 outflow spectra described in Table 1, which is the same as Table 2 in Mas-Ribas \& Mauland (2019), replicated here for clarity. These spectra are created from subsets of the total BAL catalog regarding outflow velocities (derived from the distance between the position of the minimum flux within each C IV absorption trough and the corresponding C IV emission line), velocity width of the troughs, degree of detachment between the troughs and the quasars (characterized by the minimum velocity of the troughs), and visual redshift and absolute $i$-band magnitude of the quasars. All of the parameter values are taken from the DR12QBAL catalog, for the case of the troughs. For the case of the quasar redshift and absolute magnitude, all of the values are from the DR12Q catalog, where further descriptions on their measurements are detailed. We initially consider all the troughs in the DR12QBAL catalog, for completeness, and we do not apply any cut regarding the methods for which they were measured. Because our analysis concerns the comparison between ionic species and/or subsamples, the method for measuring parameters should not have a significant impact on our results and conclusions. The number of subsamples for each parameter is generally set to be the minimum, in order to contain a large number of troughs (yielding high signal-tonoise composites) while enabling us to precisely trace the evolution of the line equivalent widths addressed in later sections. For instance, we create several subsamples with small numbers of objects at outflow velocities below $\approx 1000 \mathrm{~km} \mathrm{~s}^{-1}$ because, as we will discuss later, these velocities potentially trace halo gas and not outflows, and the measured equivalent widths for the line and line-locking components show different behaviors in this range. Other groups using our spectra can find helpful subsamples that differentiate between these velocity ranges (e.g., separating NALs and mini-BALs). Also, when possible, we apply cuts to the data, aiming to have similar numbers of objects in each bin, in order to aid comparisons between subsamples. For the case of trough width, we perform a cut at the peak of the width distribution $\left(\approx 700 \mathrm{~km} \mathrm{~s}^{-1}\right.$ in Figure 2 of Mas-Ribas \& Mauland 2019) given its sharpness, which results in one of the width bins having a lower number of troughs than the others-but without impact on the results. 
Table 1

Outflow Samples

\begin{tabular}{|c|c|c|c|}
\hline $\begin{array}{l}\text { Selection Criteria } \\
\text { all }\end{array}$ & Mean & $\begin{array}{c}n(\AA)^{\mathrm{a}} \\
0.437 \pm 0.029\end{array}$ & $\begin{array}{c}\text { No. Troughs } \\
59,872\end{array}$ \\
\hline \multicolumn{4}{|c|}{ Outflow velocity $\left(\mathrm{km} \mathrm{s}^{-1}\right)$} \\
\hline$v<200$ & 139 & $0.335 \pm 0.064$ & 185 \\
\hline$v<350$ & 264 & $0.331 \pm 0.045$ & 3167 \\
\hline$v<650$ & 315 & $0.254 \pm 0.040$ & 4355 \\
\hline $350 \leqslant v<650$ & 452 & $0.278 \pm 0.038$ & 1188 \\
\hline $650 \leqslant v<1500$ & 1122 & $0.291 \pm 0.037$ & 2278 \\
\hline $1500 \leqslant v<3000$ & 2233 & $0.387 \pm 0.081$ & 6706 \\
\hline $3000 \leqslant v<5000$ & 3980 & $0.444 \pm 0.095$ & 7727 \\
\hline $5000 \leqslant v<8000$ & 6426 & $0.462 \pm 0.031$ & 10,183 \\
\hline $8000 \leqslant v<13,000$ & 10,330 & $0.439 \pm 0.050$ & 12,488 \\
\hline $13,000 \leqslant v<17,500$ & 15,208 & $0.354 \pm 0.078$ & 9075 \\
\hline$v>17,500$ & 20,867 & $0.302 \pm 0.138$ & 10,737 \\
\hline \multicolumn{4}{|c|}{ Trough width $\left(\mathrm{km} \mathrm{s}^{-1}\right)$} \\
\hline$v<560$ & 508 & $0.227 \pm 0.045$ & 11,250 \\
\hline $560 \leqslant v<708$ & 629 & $0.264 \pm 0.094$ & 8420 \\
\hline $708 \leqslant v<1260$ & 951 & $0.305 \pm 0.149$ & 13,488 \\
\hline $1260 \leqslant v<2240$ & 1704 & $0.381 \pm 0.057$ & 10,266 \\
\hline$v \geqslant 2240$ & 5179 & $0.560 \pm 0.056$ & 20,125 \\
\hline \multicolumn{4}{|c|}{ Trough min. velocity $\left(\mathrm{km} \mathrm{s}^{-1}\right)$} \\
\hline$v<300$ & 128 & $0.423 \pm 0.048$ & 2574 \\
\hline $300 \leqslant v<2300$ & 1236 & $0.431 \pm 0.075$ & 10,713 \\
\hline $2300 \leqslant v<5300$ & 3694 & $0.381 \pm 0.132$ & 10,661 \\
\hline $5300 \leqslant v<9000$ & 7113 & $0.352 \pm 0.032$ & 10,356 \\
\hline $9000 \leqslant v<13,500$ & 11,183 & $0.400 \pm 0.082$ & 10,570 \\
\hline $13,500 \leqslant v<18,500$ & 15,953 & $0.409 \pm 0.054$ & 10,059 \\
\hline$v \geqslant 18,500$ & 21,186 & $0.463 \pm 0.089$ & 8630 \\
\hline \multicolumn{4}{|c|}{ Quasar redshift } \\
\hline$z<1.95$ & 1.77 & $0.449 \pm 0.093$ & 10,580 \\
\hline $1.95 \leqslant z<2.20$ & 2.09 & $0.455 \pm 0.047$ & 10,584 \\
\hline $2.20 \leqslant z<2.37$ & 2.28 & $0.455 \pm 0.042$ & 10,812 \\
\hline $2.37 \leqslant z<2.60$ & 2.48 & $0.414 \pm 0.066$ & 10,591 \\
\hline $2.60 \leqslant z<3.00$ & 2.79 & $0.452 \pm 0.009$ & 10,880 \\
\hline$z \geqslant 3.00$ & 3.38 & $0.461 \pm 0.159$ & 10,098 \\
\hline \multicolumn{4}{|c|}{ Quasar magnitude (mag) } \\
\hline$-25.0 \leqslant M_{i}<-22.0$ & -24.6 & $0.522 \pm 0.191$ & 10,972 \\
\hline$-25.5 \leqslant M_{i}<-25.0$ & -25.3 & $0.482 \pm 0.100$ & 10,912 \\
\hline$-26.0 \leqslant M_{i}<-25.5$ & -25.8 & $0.428 \pm 0.061$ & 12,932 \\
\hline$-26.5 \leqslant M_{i}<-26.0$ & -26.2 & $0.415 \pm 0.040$ & 12,020 \\
\hline$-27.0 \leqslant M_{i}<-26.5$ & -26.7 & $0.387 \pm 0.037$ & 8949 \\
\hline$-30.0 \leqslant M_{i}<-27.0$ & -27.5 & $0.383 \pm 0.074$ & 7749 \\
\hline
\end{tabular}

Note.

a This parameter denotes the values of the intercept in Equation (3) and is an indicator of the width of the absorption lines in the composite spectra.

Due to the shape of that distribution, the sample with the largest width range $\left(v \geqslant 2240 \mathrm{~km} \mathrm{~s}^{-1}\right)$ covers a broad range of values and has a large number of troughs, but additional cuts do not yield extra information. The samples of quasar redshift and magnitude are built considering similar number of objects per bin while evenly covering the parameter space. The first column in Table 1 denotes the cuts applied to create the corresponding subsamples, and the mean values of the respective parameters are quoted in the second column. The third column denotes the values of the intercept, which is related to the width of the absorption lines via the modeling of the absorption features described by Equation (2), and the number of spectra contributing to each subsample is presented in the fourth column. These spectra are publicly available for future outflow studies at https://github.com/lluism/BALs.

We also build and use 30 additional outflow composites, each arising from the combination of one range of trough width and one range of outflow velocity from the following respective sets: we consider five ranges of trough width, $(0,560),[560,1000),[1000,2500),[2500,5000)$, and [5000, $25,000]$, and six ranges of outflow velocity, $(0,800)$, [800, $2000),[2000,5000),[5000,10,000),[10,000,14,000)$, and $[14,000,25,000]$, where all the values have units of $\mathrm{km} \mathrm{s}^{-1}$.

The final set of outflow composites for our analysis thus consists of one spectrum built from all the troughs and 65 composites from the aforementioned subsamples. In the next section, we summarize the steps for creating these composite spectra.

\section{Methods}

We detail the methods that we use for computing the composite outflow spectra in Section 3.1, and for modeling the absorption-line profiles in Section 3.2. We briefly describe those calculations in this work, but refer the interested reader to Mas-Ribas \& Mauland (2019) and Mas-Ribas et al. (2017) for further details. In Section 3.3, we present the calculations of the equivalent widths, and we discuss measurements of the column densities in Section 3.4.

\subsection{Composite Spectra Computation}

The composite spectra are computed as a weighted mean, where the weights are derived from the signal-to-noise ratios (S/Ns) of the individual spectra, and where we use a mean quasar spectrum to remove the intrinsic quasar profile and obtain absorption-only outflow spectra.

We start by describing the computation of the mean quasar spectrum. For this calculation, we consider all the quasar spectra in the DR12Q quasar catalog, instead of only those containing broad absorption troughs, to avoid residual absorption features induced by the overlapping absorbers (see Figure 3 in Mas-Ribas \& Mauland 2019). We include here the quasars with outflow absorption troughs because they are, on average, more luminous than those without outflow signatures (e.g., median $i$-band absolute magnitude $M_{i} \sim-26.3$ mag for BAL quasars against $M_{i} \sim-25.6 \mathrm{mag}$ for the nonoutflow ones (Hamann et al. 2019)). Thus, they will contribute, in general, to the mean spectrum with weaker emission lines, due to the Baldwin effect (Baldwin 1977). These quasars correspond to $\approx 9 \%$ of the total number in the DR12Q catalog, and their average outflow absorption features imply a variation of $<3 \%$ in the mean quasar flux in the worst cases (i.e., next to the O VI and $\mathrm{N}$ V emission lines in Figure 3 of Mas-Ribas \& Mauland). The equivalent widths will be impacted by a similar fraction, which is much smaller than the uncertainties that we will compute. Overall, the inclusion or exclusion of BAL quasars in this computation has no significant impact on our conclusions.

Each quasar spectrum is shifted to the quasar rest frame, rebinned into a regular array of pixel width of $1 \AA$, and normalized by dividing it by the mean flux within two wavelength windows, $1300<\lambda_{\mathrm{r}} / \AA<1383$ and $1408<\lambda_{\mathrm{r}} / \AA<1500$, where $\lambda_{\mathrm{r}}$ denotes the quasar rest-frame wavelength. The selection of these windows enables a high $\mathrm{S} / \mathrm{N}$ for the absorption lines in the $\operatorname{Ly} \alpha$ forest, given their proximity to that region. They cover a total wavelength range large enough to obtain a reliable estimate 
of the mean flux, despite the potential contribution of the faint emission lines of O I $\lambda 1305$ and C II $\lambda 1335$. Using these windows for the composite calculation could bias the equivalent width results, as the spectra may contain the Si IV and C IV absorption troughs in them. We have compared the eleven composite spectra for outflow velocity bins in Table 1 that arise from using these windows and using the window $2600<\lambda_{\mathrm{r}} / \AA<2750$ for both the mean quasar spectrum and the composite calculation. We observe relative flux variations of $20 \%-30 \%$ between the two calculations at the peak of the strongest absorption lines (i.e., Ly $\alpha, \mathrm{N} \mathrm{V}, \mathrm{C}$ IV), and of a few percent in the unabsorbed regions and weaker absorption lines. The default window calculation results in larger fluxes. More importantly, these differences do not depend on outflow velocity-except for the subsample with outflow velocities $1500 \leqslant v<3000$, where the default window shows lower flux values by a similar amount. Overall, these differences do not impact the trends that we will observe below, so we use the default windows throughout. We also compute the mean $\mathrm{S} / \mathrm{N}$ in these windows, which we use to assign to each spectrum $j$ a weight as

$$
w_{j}=\frac{1}{\mathrm{~S} / \mathrm{N}_{j}^{-2}+\sigma^{2}},
$$

where $\sigma=0.1$ is a limitation to the contribution from very high $\mathrm{S} / \mathrm{N}$ spectra; for discussions of other values, see Mas-Ribas et al. (2017). Next, we compute the weighted mean of these normalized spectra, to obtain the final composite quasar spectrum.

The outflow composites are calculated in a similar way. Here, however, we only use the DR12QBAL spectra, and shift them all to the position of the respective absorbers, for which we use the position of the minimum flux within the absorption troughs as discussed in Sections 3.2 and 6.1 in Mas-Ribas \& Mauland (2019). We rebin the spectra into a new regular array, this time with a pixel width of $0.3 \AA$, to obtain the highest spectral resolution enabled by the BOSS spectra $\left(\approx 69 \mathrm{~km} \mathrm{~s}^{-1}\right)$. We then normalize each of them with the corresponding mean flux in the same two windows used for the mean quasar spectrum. Each normalized spectrum at the rest-frame position of the outflow (i.e., absorption trough) is then divided by the previously computed mean quasar spectrum, shifted also to the position of the absorber. This step eliminates the quasaremission profile and results in an almost flat spectrum with the outflow absorption features. The final composites are computed as the weighted mean of the absorption spectra of interest for each subsample, using the weights that were assigned to each spectrum in the mean quasar calculation.

\subsection{Line Profile Modeling}

The absorption line profiles are modeled considering the line and line-locking components of each of the transitions reported in Table 1 of Mas-Ribas \& Mauland (2019) and publicly available at https://github.com/lluism/BALs.

We first renormalize each composite spectrum, because residual contamination still exists after the composite computation. We do so by dividing the spectrum by a pseudocontinuum, obtained by smoothing the absorption-free regions of the spectrum itself with a Gaussian kernel with $\sigma_{\mathrm{G}}=10$ pixels. Despite this renormalization, the unabsorbed parts of the outflow spectra can, in some cases, not be completely flat. Therefore, we model the profiles accounting for a local continuum computed over the individual absorption features. This local continuum is obtained as a linear fit to a number of pixels in both sides of each absorption feature (for further details on these procedures, see Section 3.3.1 in Mas-Ribas \& Mauland 2019).

Next, a least-squares fit is performed to the line and linelocking components of each transition within a wavelength window containing the absorption feature, via the expression

$$
\begin{aligned}
F_{\lambda}= & C_{\lambda} \exp \left[-b \exp \frac{-\left(\lambda-\lambda_{\mathrm{c}}\right)^{2}}{2 a^{2}}\right. \\
& \left.-d \exp \frac{-\left(\lambda-\lambda_{\mathrm{ll}}\right)^{2}}{2 a^{2}}\right] .
\end{aligned}
$$

Here, $C_{\lambda}$ denotes the local continuum, and $\lambda_{\mathrm{c}}$ and $\lambda_{11}$ are the line and line-locking positions, respectively. As in Mas-Ribas \& Mauland (2019), we set a separation of $497 \mathrm{~km} \mathrm{~s}^{-1}$ between the line and line-locking components, equivalent to the distance between the two lines of the C IV doublet. Given the resolution of the BOSS spectra, however, variations of $\left|\delta_{\mathrm{v}}\right| \lesssim 30 \mathrm{~km} \mathrm{~s}^{-1}$ in the relative position of the line locking do not change the fits significantly, but we adopt the C IV value because it is physically motivated. We fit the absorption features considering a half-window size of $4 \AA$ around the center of the absorption lines, and we add an extension of $500 \mathrm{~km} \mathrm{~s}^{-1}$ on the blue side to account for the line-locking component. In cases of overlapping between multiple windows, we consider a single window, from the lowest to the highest limit of the intervening windows, and fit all the profiles simultaneously. In general, this yields the fitting of up to five absorption lines, and up to five for the range covering the $\mathrm{O}$ VI doublet. Two ranges at around 1125 and $1195 \AA$, covering ten weak features each, are split in four ranges of five lines each. We have explicitly checked that these divisions do not impact the measurements. The parameters $b$ and $d$ in Equation (2) represent the depth of the absorption features and are set free, while the parameter $a$ is related to the width of the lines and is fixed considering the spectral resolution of the BOSS spectra and its wavelength dependence. Specifically, we compute the mean values of the $a$ parameter obtained when this is set free in the various subsamples for each transition, and we use these mean values to model the slope of the evolution of $a$ with wavelength (Figure 4 in Mas-Ribas \& Mauland 2019). This calculation results in the linear fit

$$
a=(3.4 \pm 0.6) \times 10^{-4} \lambda+n,
$$

where $n$ is the intercept obtained for each subsample by fitting the above equation to the free $a$ values of the strongest transitions in each case. The values and uncertainties of $n$ for each subsample are reported in the third column of Table 1, and all parameters in the above equation have units of $\AA$. A few uncertainties for the $n$ parameter appear much larger than the others. These are produced by some $a$ parameters used for the estimation of $n$ that depart strongly from the average values. This likely reflects uncertainties in the fitting, as observed for the C IV $\lambda 1548$ line in some cases, where this line is overestimated and the line-locking C IV $\lambda 1550$ component underestimated. Details on the aforementioned calculations and 
parameters are further discussed in Sections 3.3.2 and 3.3.3 of Mas-Ribas \& Mauland (2019).

\subsection{Equivalent Width}

The equivalent width of each line and each line-locking feature in all the composites is measured by integrating the modeled absorption profile under the continuum within the corresponding absorption window. In detail, we integrate $1-F_{\lambda} / C_{\lambda}$.

\subsubsection{Equivalent Width Uncertainty}

Although the bootstrap method is suitable for calculating the equivalent width uncertainties in stacking studies (Mas-Ribas et al. 2017), the large number of spectra and samples addressed in the current work would require performing an unfeasible number (on the order of $10^{5}$ ) of spectral stacks. Instead, we derive the uncertainty of the equivalent widths from the individual normalized outflow absorption spectra, following the method detailed in Section 3.4 of Arinyo-i-Prats et al. (2018). We emphasize that this method, as well as the bootstrap, takes into account the noise from the pixels, but it does not include the systematic uncertainty from the modeling of the continua or the line profiles.

The equivalent width uncertainty for an absorption feature in one single spectrum is computed as (Equation (2) in Arinyo-iPrats et al. 2018)

$$
\sigma_{\mathrm{W}}=\bar{\sigma}_{\mathrm{t}} N_{\mathrm{p}}^{1 / 2} \mathrm{~d} \lambda
$$

where $\mathrm{d} \lambda=0.3 \AA$ is the wavelength step in the normalized outflow spectra, and $\bar{\sigma}_{\mathrm{t}}^{2}$ is the mean of the squared transmission error $\sigma_{\mathrm{t} i}^{2}=\sigma_{i}^{2} / C_{i}^{2}$ of the $N_{\mathrm{p}}$ pixels in the two ranges used for the calculation of the continuum. The calculation of the uncertainty is performed in these ranges, instead of in the line windows, to avoid possible effects due to correlations between neighboring pixels in the BOSS spectra (Arinyo-i-Prats et al. 2018). The terms $\sigma_{i}^{2}$ and $C_{i}^{2}$ in the previous expression denote the squared values of the uncertainty and continuum, respectively, for a pixel $i$.

For each feature in a composite spectrum, we then compute the mean of the squared $3 \sigma$ clipped uncertainties of the spectra that contribute to that composite. The final equivalent width uncertainties are simply the square roots of these means.

\subsection{Column Density}

In Mas-Ribas et al. (2017), we developed a theoretical model for estimating the column densities from the equivalent width of the absorption features. This model is intended for the analysis of composite spectra and it takes into account the saturation of the absorption lines. These characteristics make it useful for our current work, as absorption lines that are saturated in reality can appear weak in our composite spectra, due to the BOSS resolution, and thus the equivalent width is the only quantity that can be directly measured from the stacks (York et al. 2000).

However, the outflow material often does not cover the totality of the radiation source (partial covering), and thus the absorption lines, although saturated, do show a residual flux at the bottom of the troughs (e.g., Hamann et al. 1993; Hamann 1998; Arav et al. 1999; Borguet et al. 2012). When this happens, the equivalent widths can lead to incorrect (underestimated) column density measurements-and consequently, to unrealistic outflow parameters (Arav et al. 2013; Chamberlain et al. 2015, and references therein). Furthermore, the outflow composites contain a small number of strong transitions of each species, and the uncertainties in their equivalent widths are typically large. These two factors prevent us from accurately calibrating the model, as this requires the largest possible number of transitions for (at least) one species; see Section 6 in Mas-Ribas et al. (2017). In turn, the inferred outflow column densities are highly sensitive to the choice of the range covered by the parameter space and the values assigned to the priors in our Monte Carlo computation. We therefore decide to not attempt the measurement of the column densities in this paper, and defer it to future work where this can be addressed via numerical modeling.

\section{The Mean Outflow Absorption Spectra}

Figure 1 shows the outflow composite spectrum of all the C IV troughs in the BAL catalog ("all" sample in Table 1).

The black line in Figure 1 displays the central wavelength region of the outflow spectrum, and the pale red line shows the final composite spectrum for the "total sample" of damped Ly $\alpha$ (DLA) systems in Mas-Ribas et al. (2017) detailed below. Both spectra are normalized for comparison, using a pseudocontinuum computed by smoothing the spectra with a Gaussian kernel of $\sigma_{\mathrm{G}}=80$ pixels. This simple smoothing introduces spurious flux features that rise above unity next to some of the strongest absorption lines, and so these spectra are only valid for visualization purposes. The faint gray dashed line denotes the fraction of spectra in the BAL catalog that contribute to this composite at every pixel. This fraction decreases toward low and high wavelengths, due to the redshift of the quasars and the spectral coverage of the BOSS spectrograph.

Contrary to the case of quasar outflows that represent highly ionized material on average, the low-ionization absorption features dominate the DLA spectrum (red line) and the highionization metal lines are weak, because DLAs trace large column densities of neutral hydrogen gas in galaxies, typically self-shielded against the external radiation field $\left(N_{\mathrm{H} \mathrm{I}}>\right.$ $2 \times 10^{20} \mathrm{~cm}^{-2}$; see the review by Wolfe et al. 2005). The largest difference between the two spectra occurs for the $\mathrm{N} \mathrm{V}$ absorption doublet, followed by that of O VI, which may illustrate the impact of turbulent and/or collisional processes in the outflow. The SiIV absorption feature is weaker in the outflow than in the DLA spectrum, contrary to the other highionization doublets, which could be linked to the photoionization properties of the media inhabited by this species. We discuss these measurements in the context of the outflow structure further in Section 6.

Figure 2 displays a comparison of outflow composites for four different ranges of outflow velocities, at wavelengths below $1680 \AA$ (where most of the outflow UV absorption occurs). We have renormalized the spectra for visualization and labeled the transitions most relevant for our discussion, with the line-locking feature visible on the low-wavelength side of the strongest ones (e.g., C IV, Si IV, or Ly $\alpha$ ). Despite the noise, Figure 2 suggests that the strongest features of the doublets $\mathrm{O}$ VI and $\mathrm{N} \mathrm{V}$ appear at outflow velocities above $1500 \mathrm{~km} \mathrm{~s}^{-1}$ (red and orange lines). However, for ions such as C III, Si III, and the $\mathrm{H}$ I transitions other than $\operatorname{Ly} \alpha$, this seems to happen for the outflow velocity range between 650 and $1500 \mathrm{~km} \mathrm{~s}^{-1}$ (violet line). Also, the doublets C IV, Si IV, and the Ly $\alpha$ line 


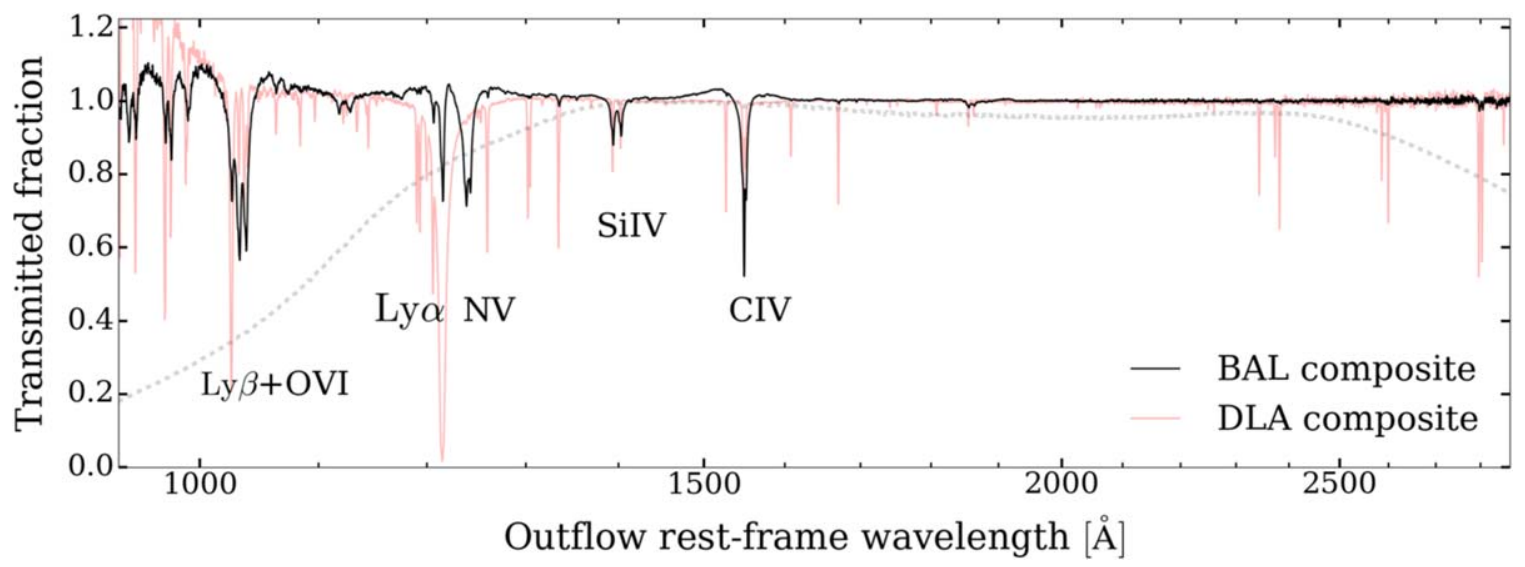

Figure 1. Outflow absorption composite spectrum, considering all the troughs in the BAL catalog (black line). Strong absorption lines of highly ionized species such as C IV, Si IV, N V, and O VI indicate a general high-ionization state for the outflowing medium-although low-ionization species are also visible, suggesting a multiphase structure. For comparison, the pale red line shows the composite spectrum of the "total sample" of DLAs in Mas-Ribas et al. (2017). This spectrum describes systems with large hydrogen column densities associated with galaxies, and thus reveals much weaker high-ionization absorption lines and stronger lowionization features compared to that of quasar outflows. The outflow spectrum is normalized for visualization using a pseudo-continuum computed by smoothing the spectra with a Gaussian kernel of $\sigma_{\mathrm{G}}=80$ pixels that results in artificial flux transmission above the unity around some of the strongest lines. This spectrum is therefore only adequate for visualization purposes. The faint gray dashed line denotes the fraction of spectra in the BAL catalog that are used for the outflow composite at every pixel, decreasing toward low and high wavelength values, due to the redshift of the quasars and the spectral coverage of the BOSS spectrograph. The horizontal axis is logarithmically spaced.

show the same strength in the red and violet lines, contrary to the previous ions. In the next section, we will show that the quantification of these observed differences suggests different phases and/or different physical processes driving these features in the outflow.

Figure 2 also shows that the average impact of the outflow absorption features in the $\operatorname{Ly} \alpha$ forest is important down to wavelengths of $\approx 920 \AA$. This effect arises mostly from the Lyman transitions of hydrogen, high-ionization species such as $\mathrm{P}$ V, O VI, and S VI, and intermediate ions such as N III and C III. At shorter wavelengths, the noise is large and the detection of lines is difficult, but additional features of S IV and a few other intermediate to high ions may be present in individual cases.

We conclude by noting that we observe a large number of high- and low-ionization absorption features in our outflow spectra, but we find no evidence for the presence of molecular $\mathrm{H}_{2}$ in any case. However, the line-locking components on the left-hand side of the absorption lines may overlap with some of the molecular hydrogen features and thus prevent their detection.

\section{Outflow Dissection}

In the following subsections, we assess the dependences of the line and line-locking equivalent widths on outflow and quasar properties. We will refer to the respective absorption components as line and line-locking from now on, for simplicity.

\subsection{Outflow Velocity Dependence}

We address here the evolution of the equivalent widths with outflow velocity, and examine the differences between ions of different species and ionization states.

The panels in the middle row of Figure 3 display the evolution of the equivalent widths for the strongest lowionization species, and those of the high-ionization species are shown in the bottom row. The measured equivalent widths are represented by colored points connected by straight lines for visualization, and their horizontal positions correspond to the values in the second column of Table 1 . The leftmost panels in the two lower rows of the figure represent the values measured for the line-lockings, and the central panels show the values measured for the lines. The rightmost panels display the total equivalent width for each species, simply adding the values of the two respective panels on their left. For guidance, we plot the uncertainties ( $1 \sigma$ values) of the line equivalent widths as vertical lines in the central panels, following the same color code as the corresponding lines and line-lockings. We emphasize again that these uncertainties are derived from the spectral pixels and do not include the possible effect of systematics or modeling (see Section 3.3.1). The left panel in the top row displays the evolution of the intercept $n$, related to the width of the absorption features through Equations (3) and (2), and the right panel represents the total equivalent width ratios between the two transitions of atomic doublets (short over long wavelength). Here, the dashed horizontal lines highlight the ratio values $2,1.5$, and 1 , which describe a saturation level increasing from nonsaturated to completely saturated absorption features. The format just described for this figure will also be used when addressing the dependences of the equivalent widths on other parameters, unless stated otherwise.

Figure 3 shows a quasi-constant equivalent width for the low-ionization species in the first $\sim 2500 \mathrm{~km} \mathrm{~s}^{-1}$ in general (middle row), although differences between the line and linelocking at the smallest velocity bins are visible. This is because the data points are located at the velocity position of the troughs in all cases, although the line-locking denotes velocities $\approx 500 \mathrm{~km} \mathrm{~s}^{-1}$ larger than those of the lines. The equivalent widths of the low-ionization lines (panel in the center) are smaller in the first $\sim 500 \mathrm{~km} \mathrm{~s}^{-1}$ than at larger velocities. This may reflect the impact to the absorption features of the gas in the quasar host halo, not in the outflow. Halo gas is less metal-enriched and moves at the typical virial velocities of 200-300 $\mathrm{km} \mathrm{s}^{-1}$ for halos of mass $M_{\mathrm{h}}$ a few times $10^{12} M_{\odot}$ at our average redshift of $z \sim 2.4$. Furthermore, stellar winds with velocities of several hundreds of $\mathrm{km} \mathrm{s}^{-1}$ could also 


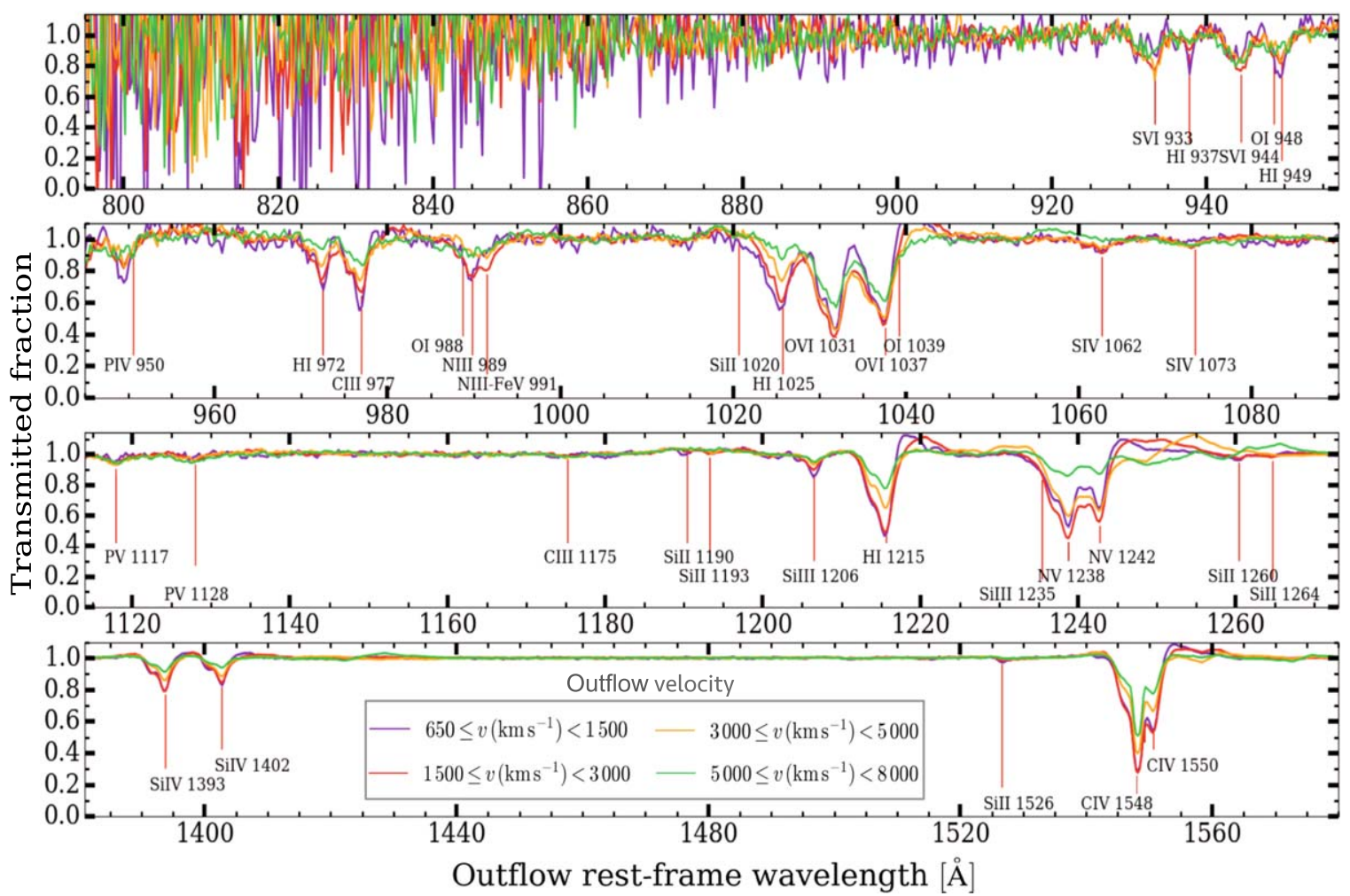

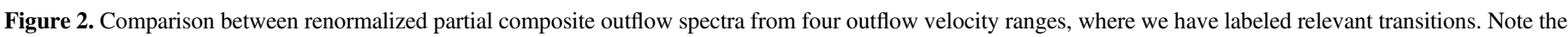

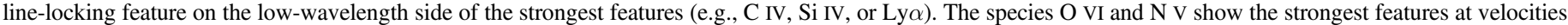

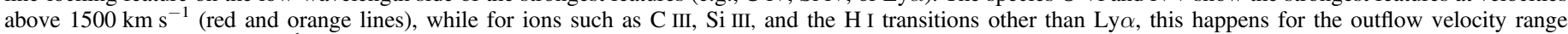

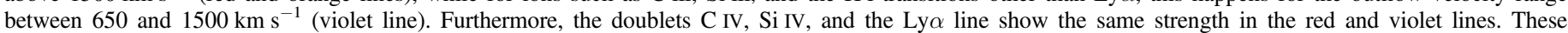

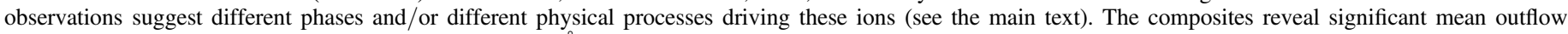
absorption features within the Ly $\alpha$ forest down to $\approx 920 \AA$, although strong lines are observed at shorter wavelengths in individual cases.

contribute to this velocity range. The equivalent widths of both low- and high-ionization species show a decrease above $2500-4000 \mathrm{~km} \mathrm{~s}^{-1}$. This decrease may denote a transition between two media with different physical properties, e.g., metal abundance or density. This could happen, for instance, in the transition region between the circumnuclear parts close to the black hole and the less dense interstellar medium, but this needs to be tested by future analysis.

The general evolution of the equivalent width for the high ions is considerably flat before the steep decrease at the same velocity as the low ions. However, a different behavior is visible for the transitions of the $\mathrm{OVI}$ and $\mathrm{NV}$ ions, which resembles the shape of the evolution of the intercept, $n$ (this is most visible when comparing the evolutions for the intercept and those in the bottom right panel of Figure 3). The equivalent widths of these two species increase steeply with velocity after $\sim 500 \mathrm{~km} \mathrm{~s}^{-1}$, before peaking and decaying quickly after $\sim 2500 \mathrm{~km} \mathrm{~s}^{-1}$. This arises from the fact that, while all the absorption features in general broaden with velocity following the evolution of $n$, their depth decreases-except for the cases of $\mathrm{OVI}$ and NV, as illustrated by Figure 2. The left panel in Figure 4 shows the derivative of the equivalent widths with respect to outflow velocity, to emphasize the different trends of the high-ionization species. A thick vertical line divides this panel into two parts with different scales. The left part covers a broader range to include the large values of C IV, while the right part zooms in by a factor of 10 . As already suggested in Figure 3 , between the velocity range $\sim 500-1000 \mathrm{~km} \mathrm{~s}^{-1}$, the highest values of the derivatives correspond to the $\mathrm{O}$ VI and $\mathrm{N} \mathrm{V}$ lines, indicating their steeper evolution compared to the other ions. We have omitted the uncertainties in this calculation for simplicity and visualization, but above $\sim 1000 \mathrm{~km} \mathrm{~s}^{-1}$, one expects the differences between the values to be of the order of the uncertainties. We discuss the origin of the $\mathrm{O}$ VI and $\mathrm{NV}$ behavior in Section 6.

The total equivalent width ratios of the doublets in the top right panel indicate that the absorption features of these doublets are a combination of highly and mildly saturated transitions, with average ratio values around 1.25 when the equivalent widths are measured with precision.

We observe a similar behavior for the equivalent widths of the line and line-locking absorption components in general. This suggests that the radiative effects, i.e., line locking, remain roughly constant and do not vary greatly with outflow velocity.

Finally, the evolutions plotted in Figure 3 also enable us to easily identify the impact of degeneracies between overlapping transitions: the magenta and purple lines in the bottom right panel show the effect of the overlapping line-locking C IV $\lambda 1550$ and line C IV $\lambda 1548$ features in the second lowest velocity bin, respectively. Overlapping and noise effects on the doublets can also be inferred from the top right panel, because the measurements of the ratios are highly sensitive to contaminants that shift their values away from the theoretical range within the values 1 and 2 .

\subsection{Trough Width Dependence}

Figure 5 illustrates the dependence of the equivalent widths on the width of the absorption troughs, following the same format as in Figure 3. A clear increase of the equivalent widths 

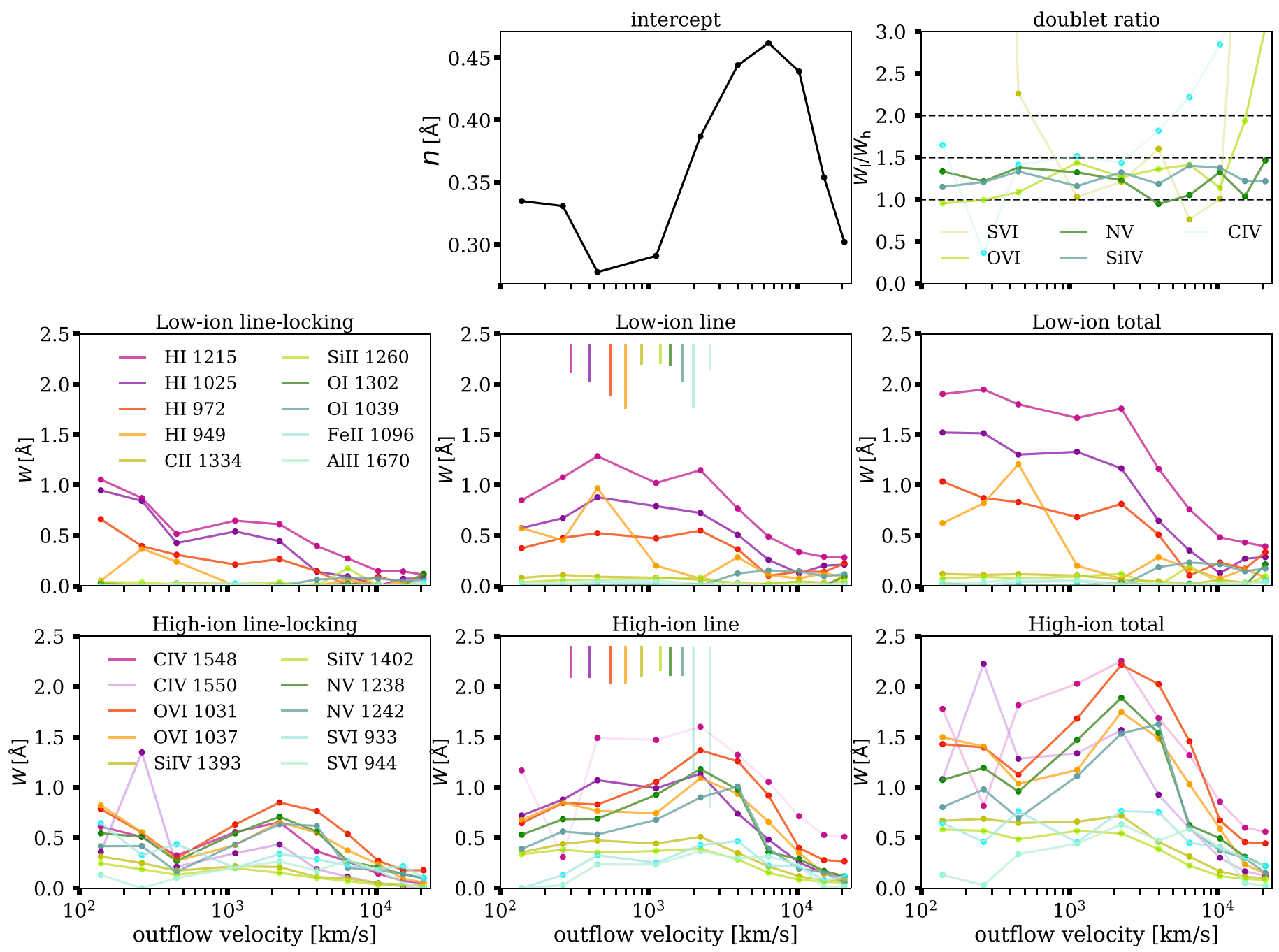

Figure 3. Evolution of the equivalent width with outflow velocity. The left panel in the top row displays the evolution of the intercept $n$, related to the width of the absorption features (Equations (2) and (3)). The ratios between the total equivalent widths of the two features of a doublet are shown in the top right panel, where the dashed horizontal lines highlight the ratio values $2,1.5$, and 1 . The panels in the middle row display the evolution of the equivalent widths for the strongest lowionization species. Those of the high-ionization species are shown in the bottom row. Measured equivalent widths are represented by colored points connected by straight lines for visualization, and their horizontal positions correspond to the values in the second column of Table 1 . The leftmost panels in the two lower rows represent the values measured for the line-lockings, and the central panels show the values measured for the lines. The rightmost panels display the total equivalent width for each species, simply adding the values of the two respective panels on their left. Uncertainties ( $\sigma \sigma$ values) of the line equivalent widths are indicated by the vertical lines in the central panels following the same color code as the corresponding lines and line-lockings. This figure shows a quasi-constant equivalent width evolution with velocity below $\sim 2500 \mathrm{~km} \mathrm{~s}^{-1}$ for both low- and high-ionization species in general, followed by a decrease at higher velocities. However, a different behavior is visible for the equivalent widths of the $\mathrm{O}$ VI and $\mathrm{N} V$ transitions, which increase steeply with velocity before the overall decrease, thus resembling the evolution of the intercept $n$ (see the text for more discussions on differences between species/transitions). Values of the doublet ratios show a combination of highly and mildly saturated lines, with a mean value at around 1.25 .

with trough width is visible, but the slope is steeper for the high-ionization species in general, and especially for the O VI and $\mathrm{N} V$ transitions, supported by the values of the derivative of the equivalent widths in the right panel of Figure 4. The equivalent width ratio values for the doublets are within the range 1-1.5, similar to those in Figure 3.

Below, we test whether the width of the troughs has an impact on the outflow velocity dependence observed in the previous section.

\subsubsection{Joint Velocity and Width Dependence}

We explore here the joint dependence of the equivalent widths on outflow velocity and trough width. This is illustrated in Figure 6, where every column denotes a range in trough width, increasing toward the right columns. The upper panels show the evolution of the intercept $n$, the middle panels chart the evolution for the equivalent widths of the low-ionization species, and those for the high-ionization species are plotted in the bottom panels.

The values of the intercept in the range of outflow velocities within $\sim 2000-6000 \mathrm{~km} \mathrm{~s}^{-1}$ present a significant increase with trough width, of up to a factor of eight for the velocity bin centered at $\approx 3700 \mathrm{~km} \mathrm{~s}^{-1}$. This trend is best matched by the high values of the $\mathrm{O} V \mathrm{VI}$ and $\mathrm{N} V$ equivalent widths at the same velocity position, compared to a smaller increase for the other ions. The values of the intercept in the minimum and maximum outflow velocity points show little evolution with trough width, indicating that the width of the lines does not change 

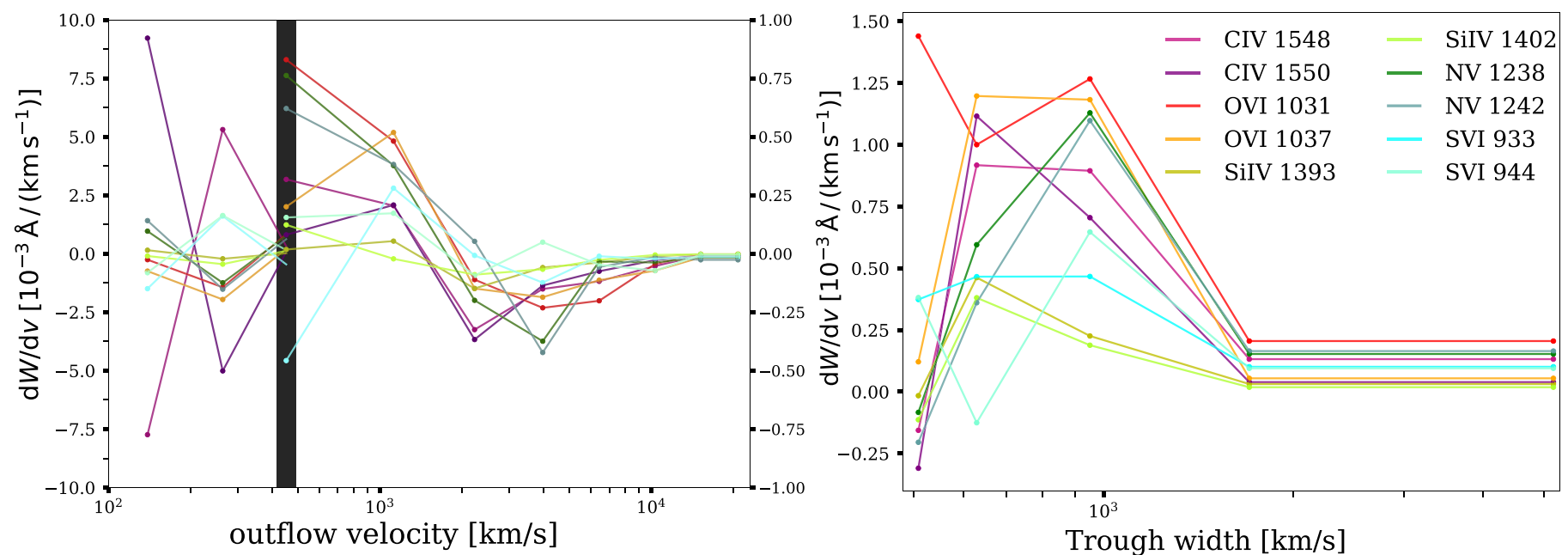

Figure 4. Left panel: evolution of the derivative of the equivalent width as a function of outflow velocity. The panel is divided by the black vertical band into two regions with different scales. Within the velocity range $500-1000 \mathrm{~km} \mathrm{~s}^{-1}$, the highest values of the derivative correspond to the $\mathrm{O}$ VI and $\mathrm{N} v$ lines, indicating their steepest evolution compared to the other ions in that region. Right panel: same as left panel, but for trough width. The highest values below the first $\sim 2000 \mathrm{~km} \mathrm{~s}{ }^{-1}$ generally correspond to the O VI and N V lines, closely followed by those of C IV. The lines of S VI show values that are are lower but generally still above those of Si IV. We have omitted the uncertainties in this calculation, for simplicity and visualization, but we expect the differences in the values to be of the order of the uncertainties above $\sim 1000 \mathrm{~km} \mathrm{~s}^{-1}$.
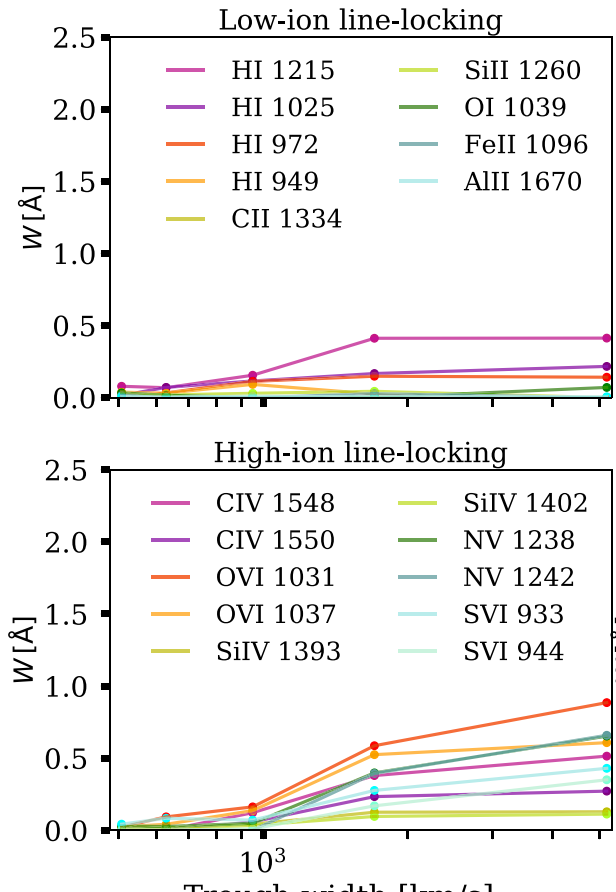

Trough width $[\mathrm{km} / \mathrm{s}]$

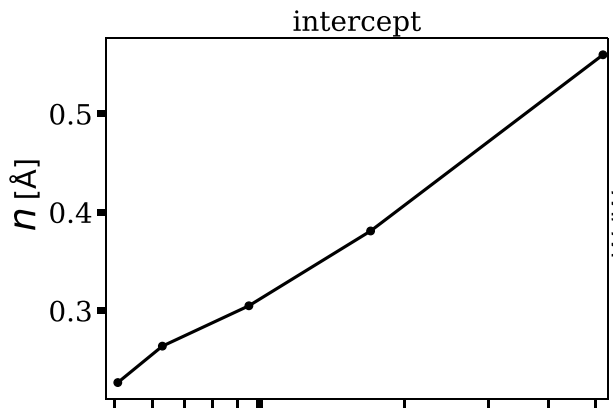

Low-ion line
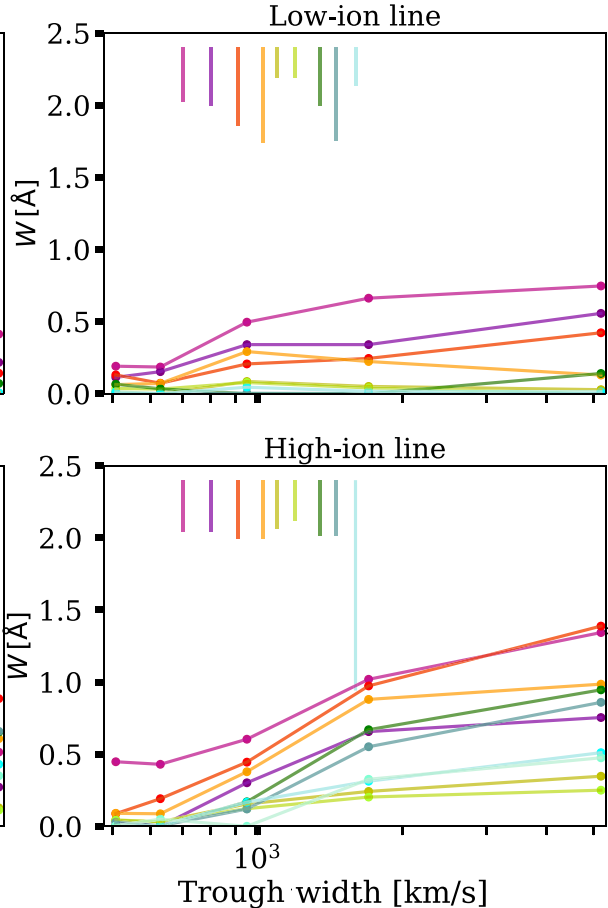
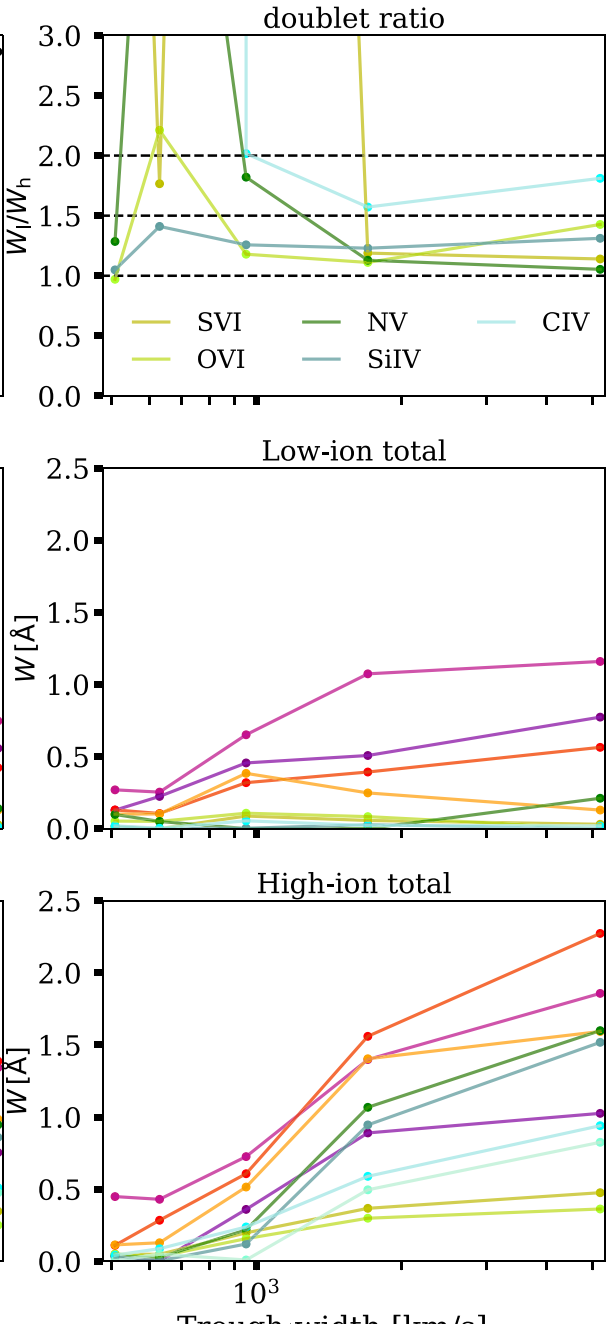

Trough width $[\mathrm{km} / \mathrm{s}]$

Figure 5. Evolution of the equivalent width as a function of trough width, following the format of Figure 3. A general increase of the equivalent width with velocity is visible, although this is steeper for the high-ionization species than for the low-ionization ones. 


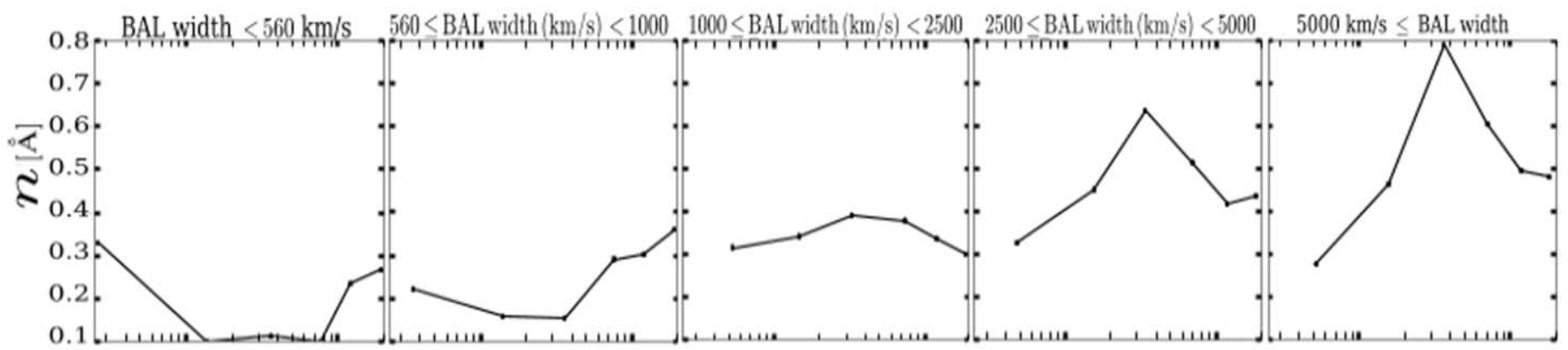

Low-ion total

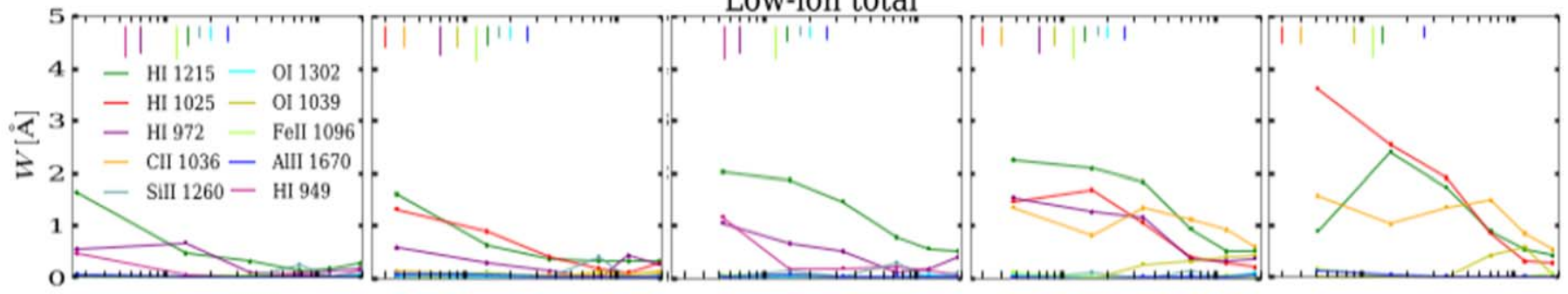

High-ion total

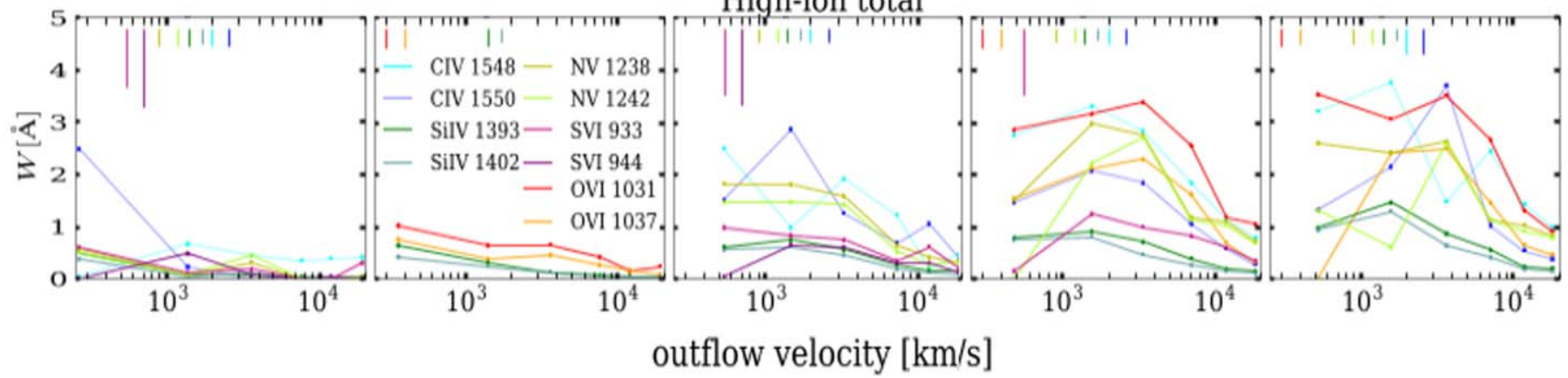

Figure 6. Evolution of the total equivalent widths of low-ionization (middle panels) and high-ionization (bottom panels) species with outflow velocity and trough width. Every column denotes a trough width range, increasing toward the right. Each top panel represents the measurements of the intercept (related to the line width) with outflow velocity, where the values in the range $\sim 2000-6000 \mathrm{~km} \mathrm{~s}^{-1}$ show a significant increase with trough width, while the values in the laterals remain almost constant. The equivalent widths of the low-ionization species increase with trough width in general, with a negative-slope dependence on velocity. High-ionization species follow a similar behavior, but the differences of O VI and N V, compared to the other ions discussed in the velocity case, are larger when increasing the trough widths.

significantly. These differences are consistent with our interpretation of three outflow velocity regions representing different gas phases or spatial locations, as discussed in Section 5.1 and also argued by Nestor et al. (2008).

In conclusion, the differences between the equivalent widths of $\mathrm{O}$ VI and $\mathrm{N} V$ compared to those of other ions are maximal at outflow velocities of a few thousands of $\mathrm{km} \mathrm{s}^{-1}$ and for troughs broader than $\sim 2500 \mathrm{~km} \mathrm{~s}^{-1}$.

Figure 6 also shows that the equivalent widths of the highionization species are generally significantly larger in the two rightmost columns than in the others, and specifically at outflow velocities above $1000-2000 \mathrm{~km} \mathrm{~s}^{-1}$. These width and outflow velocity values broadly match the strict definition of BALs (i.e., outflow velocities $\gtrsim 3000 \mathrm{~km} \mathrm{~s}^{-1}$ and trough widths $\gtrsim 2000 \mathrm{~km} \mathrm{~s}^{-1}$ ). The physical process(es) that boost the equivalent width values of some high-ionization species (see our discussion in Section 6.3) in the two rightmost panels seem to not be present in the other three leftmost columns. This effect may be the driver of the differences observed between BALs and mini-BALs/NALs, although we are here analyzing absorption lines while the outflow-type names arise from the observation of the broad troughs in the quasar spectra.

\subsection{Trough Minimum Velocity Dependence}

We assess now the dependence of the equivalent widths on the degree of detachment of the troughs, motivated by polarization results suggesting that the polarization of the outflow decreases with the velocity distance from the source (e.g., Lamy \& Hutsemékers 2004).

We show in the Appendix the dependence of the equivalent widths on the minimum velocity of the trough. The evolutions match those found with respect to the outflow velocity within the uncertainties, and additional effects arising from the detachment are not observed. This is not surprising, because our trough widths are generally narrow, with their distribution peaking at $\sim 700 \mathrm{~km} \mathrm{~s}^{-1}$ (Figure 1 in Mas-Ribas \& Mauland 2019), so the minimum trough velocities are similar to the velocities of the outflow.

We discuss the relation between the degree of polarization and the velocity of the outflows in more detail in Section 7.2.

\subsection{Quasar Magnitude and Redshift Dependences}

The Appendix shows the dependence of the equivalent widths on the absolute magnitude of the host quasars, where no 
evolution is detected. For the case of the lines, some highionization species (middle bottom panel) tentatively suggest a decrease of the equivalent width with increasing quasar brightness, consistent with the trend of the intercept (top left panel). However, this decrease is not visible for the linelocking or the low-ionization features, and the variations are at the level of the uncertainties in the measurements. We discuss the lack of dependence on the quasar magnitude and compare it with other works in Section 7.3.

The Appendix illustrates the dependence of the equivalent widths on quasar redshift. The measurements remain constant over the whole redshift range, indicating that the equivalent width values are mostly driven by local processes and are independent of the cosmic metallicity evolution. It is worth noting, however, that our measurements cover a narrow redshift range compared to the overall period of cosmic metal enrichment.

\section{The Multiphase Outflow Structure}

Figures 2 and 3, together with Figure 4, show that the dependence on outflow velocity of the O VI and N V transitions is significantly different from that seen for other low- and highionization species. The behavior of these two ions resembles that of the intercept ( $n$; top left panel in Figure 3), the latter being related to the width of the absorption features (see also the evolution of the intercept and these two species in Figure 6, and a quantitative estimate of this relation through a Spearman rank correlation analysis in the Appendix). Similar differences are also suggested in the bottom right panel of Figure 5, where the features of O VI and N V show a steeper slope compared to the other high-ionization species in the trough width range $\sim 1000-2000 \mathrm{~km} \mathrm{~s}^{-1}$, and in some cases also at larger width values. Furthermore, the bottom right panel in Figure 5 tentatively suggests that the slope of the equivalent width evolution with trough width might be steeper for C IV and S VI than for Si IV, although flatter than those of O VI and N V. This might also be the scenario illustrated in the bottom middle panel of Figure 3, supported by the values of the derivatives of the equivalent widths with trough width in the right panel of Figure 4. The uncertainties in these last cases, however, are large and the suggested trends may not be real.

\subsection{The Russian Doll Model}

We propose a multiphase stratified structure for the outflow in order to explain the different evolutions of the species, inspired by that proposed by Stern et al. (2016) for the circumgalactic medium. In the typical conditions of the circumgalactic medium, the $\mathrm{O}$ VI and $\mathrm{N} V$ species trace gas regions that are less dense and slightly hotter $\left(T \sim 2-3 \times 10^{5} \mathrm{~K}, n_{\mathrm{H}} \sim 5 \times 10^{-5} \mathrm{~cm}^{-3}\right)$ than those inhabited by CIV and S VI $\left(T \sim 1-2 \times 10^{5} \mathrm{~K}, n_{\mathrm{H}} \sim\right.$ $\left.2 \times 10^{-4} \mathrm{~cm}^{-3}\right)$, and especially by Si IV $\left(T \sim 7 \times 10^{4} \mathrm{~K}\right.$, $n_{\mathrm{H}} \sim 10^{-3} \mathrm{~cm}^{-3}$ ) on average; see Figure 6 in Tumlinson et al. (2017) and Figure 1 in Stern et al. (2016).

Figure 7 illustrates a cartoon of our proposed Russian doll model for the multiphase outflowing gas. Here, O VI and $\mathrm{N} \mathrm{V}$ trace the outermost UV parts of the outflowing material (red area), exposed to the strong radiation field (large arrow) that keeps them highly ionized. A second inner region (orangeyellow area) may then contain the C IV and S VI, where the ionizing flux has already been attenuated considerably (middle arrow) by the first UV layer, allowing a higher density and a lower temperature. Two additional regions deeper in the gas (green and light blue regions), with lower temperatures, higher densities, and less exposure to the radiation (small arrow), host the Si IV and the low-ionization species, respectively. A Si IV protected from the strong radiation field in the deepest regions of the outflowing material could explain the similarity between the equivalent width evolution of Si IV and the low-ionization species, as well as the fact that Si IV is the only high-ionization species that shows a weaker feature in quasar outflows than in DLAs in our Figure 1. However, we saw in Mas-Ribas et al. (2017) that the equivalent width of Si IV does not correlate with the column density of neutral hydrogen in DLAs (lower panels in Figure 11 there), which suggests different gas phases for Si IV and the low ions. Intermediate-ionization species, such as Al III, likely reside in the two inner regions as well, given that they often show a similar behavior to that of low ions (e.g., Filiz et al. 2014).

\subsection{The Radiation Shield}

Our outflow structure could also account for the "radiation shield" that is often invoked in radiatively accelerated outflow scenarios; see Murray et al. (1995) and Chelouche \& Netzer (2003), but cf. Baskin et al. (2013, 2014b). In brief, most of the acceleration arises from the absorption of UV radiation, but if the gas is highly ionized, it is too optically thin to the UV photons and the radiative acceleration becomes inefficient (the so-called overionization problem; see Castor et al. 1975, Stevens \& Kallman 1990, Higginbottom et al. 2014, and Dannen et al. 2019). Therefore, the high-energy (X-ray and farUV) photon flux needs to be significantly suppressed before reaching the acceleration region, to keep the medium optically thick to UV radiation (Leighly 2004; Baskin et al. 2013), which can be achieved with a large column density, above $N_{\mathrm{H}} \sim$ $10^{22}-10^{23} \mathrm{~cm}^{-2}$, of highly ionized material (Murray et al. 1995). This shield is sometimes assumed to have a constant density and to remain static close to the source of radiation, but Hamann et al. (2013) indicated that absorption signatures from this gas at very low velocities are not observed; see also an extended discussion in Section 5.1 of Hamann et al. (2019). Additionally, Chartas et al. $(2002,2003)$ detected broad X-ray absorption features at velocities $\gtrsim 0.2-0.3 c$ that disfavor the static medium. We suggest that the radiation-shielding gas is comoving with the outflow-or more precisely, that the radiation shields are the most external layers in the multiphase structure of Figure 7 (leftmost red and purple areas), where $\mathrm{X}$-ray and far-UV transitions take place; see McKernan et al. (2007) and Reeves et al. (2013), but cf. Hamann et al. (2018). Proga et al. (2000) found that the shield arises naturally in a disk wind scenario; see also the radiative transfer simulations in a clumpy outflow by Matthews et al. (2016).

Baskin et al. (2014b) argued that a stratified multiphase structure, such as the one proposed here, could circumvent the overionization problem without the need for a static shielding layer (see also de Kool \& Begelman 1995; Arav et al. 2013), and a structure with different phases was also suggested by Baskin et al. (2014a) and Stern et al. (2014) to explain observed properties of the BLR in active galactic nuclei (AGNs).

\subsection{The Collisional Processes}

With this outflow structure in mind, we argue that the equivalent width differences observed between species result from, and reveal, the effect of collisional processes. Collisions 


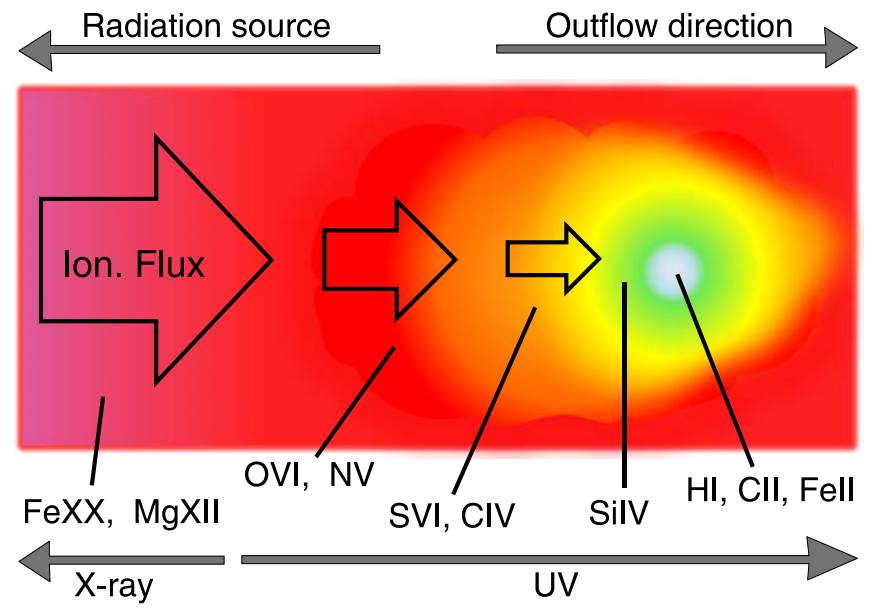

Figure 7. Cartoon of the proposed multiphase structure of the gas in outflows. From the outer to the inner regions, the ionizing radiation indicated by the arrows is attenuated and the gas has a lower temperature and higher density, enabling it to host low-ionization species in the core.

would be most important in the external layers where the temperatures are the highest-and therefore more visible for the $\mathrm{O}$ VI and $\mathrm{NV}$ ions. The presence of collisions is further supported by the detection of the $\mathrm{S} \mathrm{IV}^{*} \lambda 1073$ and $\mathrm{C} \mathrm{III}{ }^{*} \lambda 1175$ absorption lines (the latter at a considerably lower significance, as visible in Figure 2), because both arise from excited metastable states populated through collisional processes (Leighly et al. 2009). However, we only detect these transitions in the outflow spectra where the $\mathrm{O}$ VI and $\mathrm{NV}$ equivalent widths are significantly large. This is illustrated in the upper panel of Figure 8, where the excited-state transition $\mathrm{S} \mathrm{IV}^{*} \lambda 1073$ is mostly detected in the two subsamples with mean outflow velocities $\sim 2200 \mathrm{~km} \mathrm{~s}^{-1}$ (red line) and $\sim 4000 \mathrm{~km} \mathrm{~s}^{-1}$ (orange line). The lower panel in Figure 8 quantifies these observations through the equivalent width of the two sulfur lines, compared to the scaled values of the other high ions with outflow velocity (the gray lines are the same as in the bottom-right panel of Figure 3, reduced by a factor of five). Because these lines are very weak and our fitting algorithm does not perform reliably in this case, we compute the equivalent widths by summing the flux in the composite spectrum (with positive sign for flux below the unity and negative above). For this measurement, we use the same windows as those for the equivalent width uncertainty calculations in Section 3.3.1. The uncertainties in the equivalent widths are also obtained as in Section 3.3.1, but in this case we extract them directly from the stacked spectrum instead of all the intervening individual spectra. Because our uncertainty estimation does not capture the effect of systematics, such as the calculation of the smooth continuum for the final normalization of the spectrum, we consider the uncertainties in Figure 8 to underestimate the true ones. Therefore, only the large positive equivalent width values should be considered for interpreting the results of Figure 8. The $\mathrm{S} \mathrm{IV}^{*}$ and $\mathrm{C} \mathrm{III}{ }^{*}$ features are also visible in the two subsamples with mean trough widths of $\sim 1700 \mathrm{~km} \mathrm{~s}^{-1}$ and $\sim 5200 \mathrm{~km} \mathrm{~s}^{-1}$ (see Table 1).

We note that collisional processes and shocks have also been inferred recently in the circumnuclear regions of the AGN host galaxy NGC 3393 by Maksym et al. (2019) (see also references therein), based on the analysis of X-ray and optical
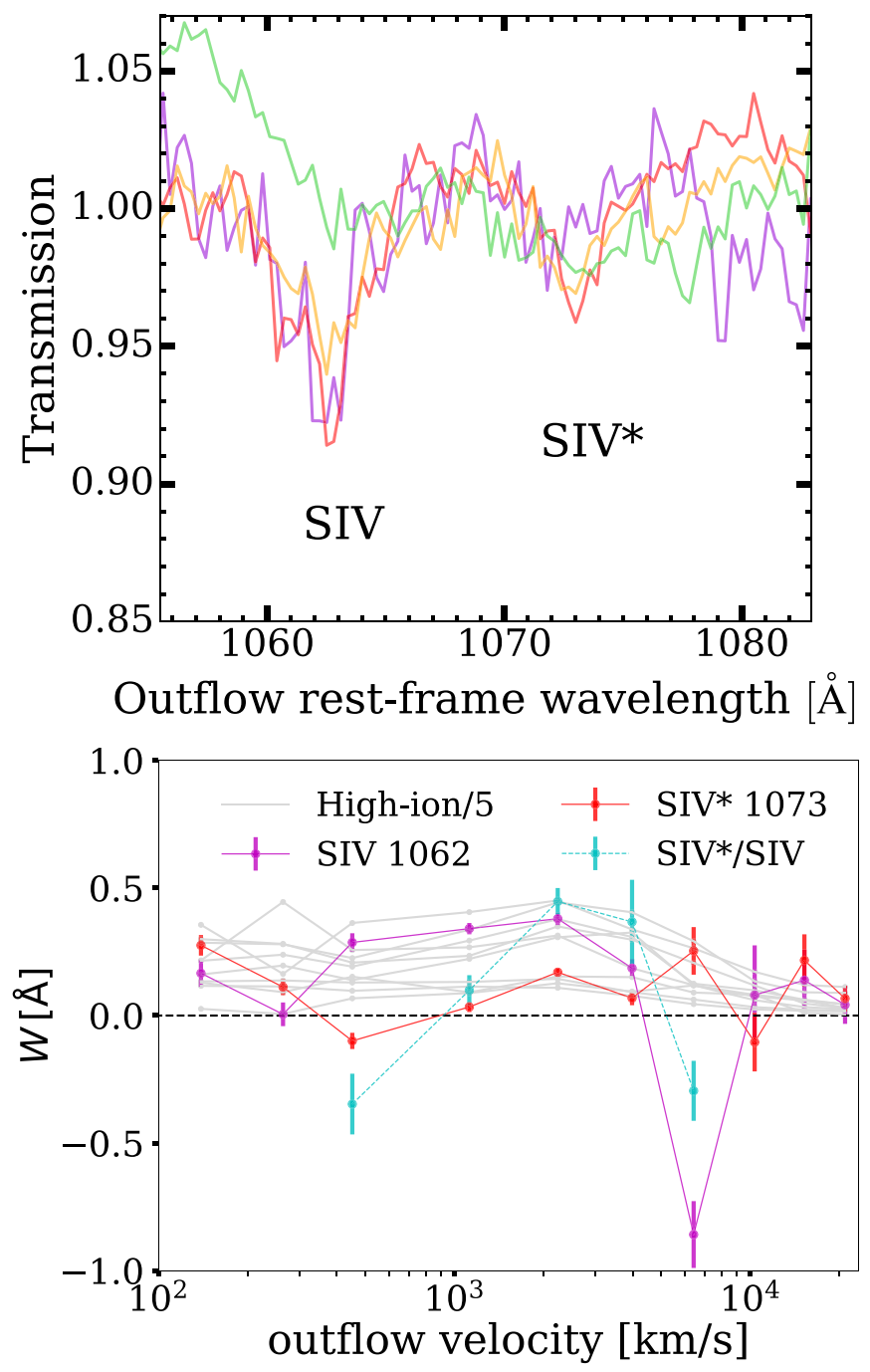

Figure 8. Top panel: rest-frame outflow wavelength range illustrating the absorption features of the ground- and excited-state transitions S IV $\lambda 1062$ and $\mathrm{S} \mathrm{IV}^{*} \lambda 1073$, respectively, for different samples of outflow velocity. The transition $\mathrm{S} \mathrm{IV}^{*} \lambda 1073$ is the strongest in the same two velocity bins (red and orange lines) where $\mathrm{O}$ VI and $\mathrm{N} \mathrm{V}$ are also the strongest, while barely visible at lower and higher velocities. Note the tentative presence of the $\mathrm{C}$ IV line-locking feature of S IV $\lambda 1062$ at $\approx 1060 \AA$ in the two strongest cases. Bottom panel: equivalent width with outflow velocity for the two sulfur transitions (violet and red lines). Blue dots and dashed line denote the values of the equivalent width ratio of the two transitions. In the central velocity range, where the two lines are well-measured, the ratio increases with velocity and peaks at a value of $\approx 0.4$. This suggests an evolution of the electron density correlated with that of the equivalent widths of $\mathrm{O}$ VI and $\mathrm{N} \mathrm{V}$, although systematic uncertainties not captured by our computations can be important.

observations at high spatial resolution of the innermost parts of this galaxy.

\subsection{The Pressure Waves}

The detection of $\mathrm{S} \mathrm{IV}^{*} \lambda 1073$ and $\mathrm{C}$ III* $\lambda 1175$ implies high electron densities in the outflow, with values typically above $n_{\mathrm{e}}=10^{3}-10^{4} \mathrm{~cm}^{-3}$ resulting from the S IV lines (Chamberlain et al. 2015; $\mathrm{Xu}$ et al. 2019), and as high as $n_{\mathrm{e}} \gtrsim 10^{9} \mathrm{~cm}^{-3}$ when C III* is also detected (Bromage et al. 1985; Kriss et al. 1992). The value of the density is derived from the column density ratios between the excited and ground state transitions of each species, i.e., S IV $\lambda 1062$ and C III $\lambda 977$, respectively. We defer detailed calculations of the electron density to future 
work, but perform a rough estimate of the values expected from our measurements following the discussion in Section 2 of the recent paper by Arav et al. (2018).

For the cases in which we reliably detect both S IV transitions (between 1000 and $5000 \mathrm{~km} \mathrm{~s}^{-1}$, where both lines show positive equivalent width values), the strength of the $\mathrm{S} \mathrm{IV}^{*} \lambda 1073$ line is smaller than that of S IV $\lambda 1062$ (lower panel in Figure 8), with observed ratios within the range $\sim 0-0.4$. The ratio values indicated as the blue dots and dashed line increase in this narrow velocity range, with outflow velocity and peak at the same velocities where we find the maximum equivalent widths of O VI and NV (red and orange lines in Figure 8). However, the uncertainty of the point at $\sim 4000 \mathrm{~km} \mathrm{~s}^{-1}$ is large, and the negative values of the ratio at the largest and smallest velocity points are unphysical and likely driven by systematics, complicating a clear interpretation. Broadly comparing our ratio values with those illustrated in Figures 1 and 2 in Arav et al. (2018) (see also Chamberlain et al. 2015), we estimate that the electron density is $n_{\mathrm{e}}<0.5 \times 10^{4} \mathrm{~cm}^{-3}$ at low outflow velocities $\left(\lesssim 1000 \mathrm{~km} \mathrm{~s}^{-1}\right)$, increasing up to almost $n_{\mathrm{e}} \sim$ $10^{5} \mathrm{~cm}^{-3}$ at $\sim 2000-4000 \mathrm{~km} \mathrm{~s}^{-1}$.

Our measurements thus suggest a correlation between the electron density (when this can be measured well), the equivalent widths of the O VI and N V transitions, and the width of the absorption lines as traced by the intercept. This result is consistent with our interpretation that collisions drive the equivalent widths of $\mathrm{O}$ VI and $\mathrm{NV}$, and that they likely also impact those from other species. Furthermore, the increase of density with velocity before the equivalent-width turnover suggests a scenario where the outflow sweeps up and compresses and transports material while moving outward, creating a pressure wave with higher densities at higher speeds (perhaps in the form of a shock wave for supersonic velocities). In the densest wave fronts, which may take the shape of sheets or filaments, the gas protected from the radiation field might cool, and thus result in the stratified Russian doll structure proposed above, which hosts low ions in its core. The decrease of density at the largest velocities could again represent a change in the properties of the medium and/or the dissipation of the waves, but this needs to be tested with numerical calculations; see the calculations of the formation, acceleration and destruction of gas clumps in outflows in Proga \& Waters (2015) and Waters \& Proga (2016), as well as the shock-wave scenario proposed for FeLoBALs by Faucher-Giguère et al. (2012).

Given the similar evolution observed for the equivalent widths of the line and line-locking absorption components in all cases, together with the omnipresence of radiativeacceleration effects that we detected in Mas-Ribas \& Mauland (2019), we postulate that radiation is the main driver of the pressure waves. Waves in quasar outflows where radiation pushes matter outward are indeed expected, based on the theory of radiatively accelerated winds; see Feldmeier \& Shlosman (2002), Dyda \& Proga (2018), and references therein, and see also Abbott (1980) and Sundqvist et al. (2018) for the case of stellar winds. A wavy behavior was already found in the disk wind simulations by Proga et al. (2000; see also Dyda \& Proga 2018). More recently, the general relativistic magnetohydrodynamical simulations with radiation transfer by Davelaar et al. (2018), although representing distances of a few gravitational radii from the black hole, also show the strong impact of radiation to the formation of wavy/filamentary structure in the outflows. Finally, a scenario in which quasar radiation accelerates material outward and creates filamentary structure similar to ours was investigated by Scoville \& Norman (1995). These authors considered that the stellar winds of massive stars orbiting the supermassive black holes would release trails of dust that would then be accelerated outward by the quasar radiation. The results by Scoville \& Norman (1995) were consistent with several observed outflow properties, such as the terminal velocities, gas column, and electron densities, and the same methodology can be applied to the acceleration of gas instead of dust. Future analysis should also explore the potential contribution from other sources of pressure, such as thermal (Chelouche \& Netzer 2003) and magnetic energy (de Kool \& Begelman 1995; Everett 2005), or cosmic rays (e.g., de Kool 1997).

Interestingly, Hamann et al. (2019) recently argued that LoBALs show the largest average column densities and highest velocities in the outflows. This result is consistent with our findings that larger velocities drive higher densities, which also relates to the presence of low-ionization species. High average densities in our proposed outflow structure could enhance the total amount of low-ionization gas, but would also increase the strength of absorption features from the high ions. This agrees with the high degree of reddening observed for LoBAL quasars (Reichard et al. 2003; Ganguly et al. 2007; Dai et al. 2012), and with the measurements by Allen et al. (2011), Reichard et al. (2003), Filiz et al. (2014), and Hamann et al. (2019), where LoBALs show absorption troughs of high-ionization species that are (at least) as large as those in HiBALs. Further potential connections between the structure of the outflows and the nature of LoBALs need to be addressed in future studies.

Finally, the value of the electron density is often used to infer the distance between the absorbing material in the outflow and the radiation source-although see the different methods discussed in Section 7.1 of Arav et al. (2018). Assuming that the conditions of the typical outflow considered by Arav et al. (2018) are similar to our measurements, a comparison with the electron densities in their Figure 2 suggests that our spectra in the range of velocities where the metastable transitions are detected traces the outflow at distances $\gtrsim 100 \mathrm{pc}$ from the radiation source. This result is consistent with the recent findings by Arav et al. (2018) and those presented by the same group in the works of, e.g., Borguet et al. (2013) and Xu et al. (2018, 2019).

\section{Discussion}

The potential impact of collisional processes on the radiative acceleration is discussed in Section 7.1, and correlations between our results and those from polarization studies are presented in Section 7.2. We discuss the lack of correlation between the equivalent widths and the magnitude of the quasars in Section 7.3, and highlight some caveats and limitations in Section 7.4. We conclude by mentioning the applicability of our findings for future work in Section 7.5.

\subsection{Collisions Suppressing Radiative Acceleration?}

An important effect of collisions on the species in the outermost UV layer of our outflow model might be related to the lack of radiative acceleration from $\mathrm{O}$ VI and $\mathrm{N} \mathrm{V}$ that we inferred in Mas-Ribas \& Mauland (2019). Our results there showed that the absorption features of these two ions had a 
strength similar to that of C IV, indicating that their optical depths - and in turn, the amounts of absorbed (i.e., scattered) radiation - are large, but their line-locking signatures revealing acceleration were not detected. It is possible that the radiative acceleration experienced by these ions from the momentum injected via the absorption and emission of photons is counterbalanced by Coulomb collisions between the metal ions and the free particles in the gas, specifically protons and electrons from ionized hydrogen.

Baskin \& Laor (2012) quantified this effect in the BLR of AGNs, and their fiducial calculations indicated that the outflow needs to be exposed to a large photon flux for the radiative acceleration to dominate over the collisions. Because the distance from the source is larger for the BALs than for the BLR, it is plausible that the collisions suppress the line locking in our case. However, if this is the reason for the nondetection of $\mathrm{O}$ VI and $\mathrm{N} V$ line locking, the question that promptly arises is what prevents CIV from suffering the same fate. The temperature of the region where C IV resides can be certainly lower than that of the two ions, and the density larger than in the highly ionized gas, but these seem to be small differences compared to the largely distinct consequences. Future work needs to investigate this processes in more detail, as well as confirm our inferred lack of radiative acceleration from the high ions.

\subsection{Correlations with Polarization}

Polarization observations in BAL quasars and outflows show that there are large differences from object to object, but some trends have been observed for the average populations (e.g., DiPompeo et al. 2010, 2013). For example, the polarization at the absorption troughs generally is larger than that of the quasar continuum and emission lines (Ogle et al. 1999). This confirms that scattering of photons in outflow scenarios is important, as scattering polarizes the radiation. Furthermore, Lamy \& Hutsemékers (2004) performed correlation and principal component analyses to optical spectra and optical polarization data from a set of 139 BAL quasars. They found that BAL troughs that start close to the emission lines (small outflow velocities) are more polarized than those that start far from the emission (detached), with a large detachment implying a large angle between the line of sight toward the observer and the accretion disk. The anticorrelation between polarization and detachment was also suggested by DiPompeo et al. (2013), but these authors argued against the viewing angle dependence being the unique reason for the decrease in the polarization.

Considering these polarization results and our findings, it is worth noting a potential physical relation between the outflow gas density and the degree of polarization. It is possible that the decrease in polarization arises from the increase in the optical depth of the outflowing gas with velocity. An optically thin medium results in a small number of scattering events, which produces a significant level of overall polarization in the scattered flux. However, when the density increases, the photons undergo many more scattering events, enabling a broad range of individual polarization values that average to a small total degree of polarization (e.g., Kim et al. 2007). This relation between the column density and the polarization in AGNs has been shown in the numerical radiative transfer calculations of Lee \& Blandford (1997) and Wang et al. (2007); see also Wang et al. (2005) and Chang et al. (2015, 2017). However, the scenario suggested here implies that the polarization arises from resonant-line scattering in the outflow. This is in conflict with some models where polarization is driven by scattering of free electrons that inhabit regions separated from the outflowing wind (e.g., Lamy \& Hutsemékers 2004, and references thereafter).

\subsection{On the Noncorrelation of Equivalent Width and Quasar Brightness}

In Section 5.4, we found no correlation between the equivalent widths of the absorption features and the absolute quasar magnitude (Figure 10). This may be not surprising, as observations indicate that the brightness of the quasars regulates the maximum velocity reachable by the outflowsbut not the minimum (Ganguly et al. 2007). If the range of magnitudes in our analysis enables similar maximum velocities, then our composites could result from samples with the same distribution of outflow velocities, and differences due to magnitude would not be visible in their average values. Indeed, we find no correlation between outflow velocity and quasar magnitude in our BAL catalog data.

The fact that the brightness of the quasars sets only the maximum outflow velocity may support the idea that the exact value of the gas velocity depends more on the physical properties of the outflow than those of the radiation source, as long as the latter provides enough photon flux to radiatively accelerate the material (Ganguly \& Brotherton 2008).

We conclude by also noting a lack of correlation between equivalent width and X-ray flux that has recently been inferred by Chartas \& Canas (2018) in a narrow-line Seyfert galaxy previously analyzed by Parker et al. (2017).

\subsection{Caveats and Limitations}

We have applied the same methodology throughout to consistently compare our stacked spectra. However, potential individual departures from the average behavior identified during our analysis are highlighted here. We stress that these effects do not impact our current results or interpretations.

In the evolution of the equivalent width with trough width (Section 5.2), some lines appeared shifted from their expected positions. This happens in the two composite spectra with the lowest average trough widths. There is no clear dependence on the ionization stage, but the fits are slightly better if we consider for these lines an offset of $30-40 \mathrm{~km} \mathrm{~s}^{-1}$ from the others (note that our pixel resolution is of $\sim 70 \mathrm{~km} \mathrm{~s}^{-1}$ ). In the same spectra, the absorption features of $\mathrm{N} \mathrm{V}$ and O VI appear narrower than that of $\operatorname{Ly} \alpha$, and Si IV shows width values in between. These differences disappear, and all transitions have a similar width, at larger trough width bins. In the evolution with outflow velocity (Section 5.1) and for the largest velocity bins, the width of $\mathrm{N} \mathrm{V}$ and $\mathrm{O}$ VI seems to be larger than for other ions. All these effects are likely due to the different impact of collisions on the different ions, which needs to be assessed in more detail in future work.

We emphasize the similar shape between the evolution of the C IV equivalent width with outflow velocity (bottom right panel of Figure 3 ) and the distribution of the number of C IV troughs with outflow velocity in the BAL catalog (upper panel of Figure 2 in Mas-Ribas \& Mauland 2019). We believe that the velocity distribution of absorption troughs in the catalog may be driven (biased) by the observability of the troughs, given the dependence of the strength of the absorption features on velocity. This 
distribution would simply reflect the difficulty of detecting C IV at a given velocity, but it would not represent the actual number of absorbers at that velocity value. In other words, we cannot know how many absorbers inhabit each velocity bin, because their detectability also depends on velocity.

\subsection{Future Work}

We briefly discuss here the impact and possible applications of our results on future outflow studies, as well as analyses that can be performed by using our public composite spectra.

1. Photo- and collisional-ionization modeling. These calculations are necessary to measure column densities and metallicities, and subsequently obtain more refined values for the densities, distances between the different parts of the outflows and the sources, and the energetics and mass loading in the outflows.

2. Detailed absorption-line fitting. Following the discussion in the previous section, a detailed examination of the differences between the width and the offset of the absorption features from different ions could reveal important kinematic information for various phases and species, as well as correlations between the kinematic and physical outflow/quasar properties. These differences would also yield more information about the impact of collisions on different ions.

A careful absorption-line search may also result in the detection of molecular hydrogen $\mathrm{H}_{2}$. Because we estimated outflow distances of a few hundreds of pc from the radiation sources, this detection would be of great importance for constraining the connection between the quasar outflows and AGN feedback (e.g., King \& Pounds 2015; Harrison et al. 2018).

3. Observations. We showed that the largest equivalent widths for high-ionization transitions occur in outflows with velocities of a few thousands of $\mathrm{km} \mathrm{s}^{-1}$, and below this value for the low-ionization species, on average. Below $\sim 500 \mathrm{~km} \mathrm{~s}^{-1}$ and above $\sim 10^{4} \mathrm{~km} \mathrm{~s}^{-1}$, the observations might be contaminated by the host galaxy. The equivalent widths of both high- and low-ionization lines increase with trough width. Therefore, broader troughs will typically show stronger features and will enable detecting the faintest associated absorption components. This may be important, for example, for the search and accurate measurements of dust indicators (e.g., absorption features from chromium) and for informing observations that search for molecular gas in outflows, such as those currently performed with the Atacama Large Millimeter Array (e.g., García-Burillo et al. 2016; Bonzini et al. 2017; Imanishi et al. 2018; Izumi et al. 2018; Spilker et al. 2018).

4. Radiation and magnetohydrodynamic simulations. An important byproduct of our work is that our results can be used as a bench test for numerical simulations. Modern simulations account for many of the physical processes involved in quasar outflows, but the lack of constraining observational information enables a large number of plausible simulated scenarios (e.g., Ciotti et al. 2010; Choi et al. 2015; Weinberger et al. 2017). Furthermore, most simulations focus either on the processes at scales of the order of a parsec around the black holes (e.g., Williamson et al. 2018) or at scales beyond the kpc where the outflows interact with the interstellar medium. The same computations also typically concentrate their numerical power either on radiative transfer or hydrodynamical processes, and only recently have they started to simultaneously incorporate both aspects (Higginbottom et al. 2013; Matthews et al. 2016; Dyda et al. 2017; Proga et al. 2017; Dannen et al. 2019, and references therein) and consider a broad range of spatial scales (see the detailed introduction by Barnes et al. 2018). The results presented in our current work, as well as in MasRibas \& Mauland (2019), concern the outflowing media inhabiting the spatial scales in between those just mentioned, and where the coupling between radiation and matter is of great importance. Therefore, the advance of numerical simulations in these regimes, informed by our results, can constrain the number of valid simulated alternatives and bridge the gap between quasar outflows and AGN feedback. Reciprocally, simulations are also needed to accurately test our interpretations and predictions, such as as those for polarization, radiation shields, orientation, shocks and radiation pressure, etc., as well as those from other studies.

\section{Conclusion}

We have analyzed 66 quasar outflow composite spectra that extend the 36 previously computed for the study of line-locking signatures in Mas-Ribas \& Mauland (2019). The composites are built from splitting the overall absorption trough data from the twelfth data release of the SDSS-III/BOSS quasar catalog in subsets of outflow velocity, width of the absorption troughs, detachment between the absorption troughs and the emission sources, and quasar magnitude and redshift. We have measured the equivalent widths of the line and line-locking components of more than 100 absorption features in each spectrum (Section 3), and have assessed the dependences of the equivalent widths on the aforementioned outflow and quasar parameters (Section 5).

The 36 outflow composites and the atomic data for absorption lines are publicly available at https://github.com/ lluism/BALs.

Our findings can be summarized as follows:

1. The outflow absorption spectra are characterized by strong features of high-ionization species, but those of low-ionization elements are also present in all cases. Despite the clear detection of many low (neutral) ions, we do not identify features of molecular hydrogen, $\mathrm{H}_{2}$, although these might be masked by the line-locking components of the absorption lines. All high-ionization absorption features are stronger in the overall outflow composite than in that of DLAs, except for the features of the Si IV doublet (Section 4 and Figure 1).

2. In general, the equivalent widths of both low- and highionization absorption features remain fairly constant with outflow velocity in the first $\sim 2500 \mathrm{~km} \mathrm{~s}^{-1}$, and decrease rapidly at higher velocities. However, the transitions of the O VI and N V doublets show a different behavior: their equivalent widths raise steeply with outflow velocity and peak at $\sim 2500 \mathrm{~km} \mathrm{~s}^{-1}$, before the general decrease. The evolution of these two ions matches that of the parameter we use to estimate the width of the absorption lines (Figure 3).

3. The equivalent width of all the species increases with trough width, but the evolution is the steepest for $\mathrm{O}$ VI and $\mathrm{N} \mathrm{V}$ (Figure 5). 
4. The maximum difference between the equivalent widths of $\mathrm{O} \mathrm{VI}$ and $\mathrm{NV}$ and those of other ions occurs at outflow velocities of a couple of thousands of $\mathrm{km} \mathrm{s}^{-1}$ and for absorption troughs of similar width values. The outflow velocity bins $\leqslant 800 \mathrm{~km} \mathrm{~s}^{-1}$ and $\geqslant 14,000 \mathrm{~km} \mathrm{~s}^{-1}$ show little equivalent width evolution with trough width, suggesting that these velocities may trace media other than the outflow, i.e., the host halo gas, and/or a transition between two physically distinct gas regions (Figure 6).

5. We find no correlation between the equivalent widths and quasar absolute magnitude or quasar redshift, consistent with the findings from other works (Section 5.4).

6. The equivalent width ratios between the two lines of high-ionization atomic doublets show average values around 1.25 in all the subsamples, implying that the composites consist of a combination of strongly and mildly saturated lines.

7. We propose a multiphase stratified "Russian doll" structure for the outflows, where deeper layers are denser, colder, and less affected by the radiation field. This structure enables us to consistently explain all the aforementioned differences observed for the equivalent width evolution of the different ions, as well as the behavior of Si IV in quasar outflows and DLAs. It also naturally accommodates the origin of the radiation shield that is often invoked for radiative acceleration in outflows (Figure 7 and Sections 6.1, 6.2).

8. Detections of transitions from collisionally populated metastable excited states in some of the outflow velocity stacks reveal the presence of collisional processes. We argue that collisions are the drivers of the equivalent width differences observed between ions, as the strength of these metastable-level features correlates with the equivalent widths of $\mathrm{O} \mathrm{VI}$ and $\mathrm{N} \mathrm{V}$ and the average width of the absorption lines (Section 6.3 and Figure 8).

9. We also infer a correlation between the electron density, the strength of the metastable-level transitions, and the outflow velocity, which we interpret as the signature of pressure waves that sweep up and compress material outward along with the movement of the outflow. Given the ubiquity of the radiative-acceleration signature found in Mas-Ribas \& Mauland (2019) and the similar evolutions observed here for the line and line-locking absorption components, we argue that radiation pressure is the main driver of the waves. The pressure-wave scenario fits well in our proposed outflow structure and it can explain the formation and survival of low-ionization species in dense parts of the wave fronts (Sections 6.3, 6.4).

10. We estimate that outflow gas with velocities of $\sim 2000-4000 \mathrm{~km} \mathrm{~s}^{-1}$ traces distances of above $100 \mathrm{pc}$ from the radiation sources.

Our proposed outflow structure can qualitatively account for the differences that we have observed in the equivalent width evolutions, but it may have implications in other results. For instance, we discussed the possible relation between the increase of density and the decrease of polarization in the absorption troughs with velocity (Section 7.2). Furthermore, the collisional scenario could be related to the lack of linelocking signatures from doublets other than CIV that we inferred in Mas-Ribas \& Mauland (2019) (Section 7.1). However, these and other interpretations need to be investigated in more detail in future work. For this purpose we have made our main composite spectra publicly available, and we have highlighted aspects that can be addressed with photo- and collisional-ionization modeling, as well as with radiation and hydrodynamic simulations (Section 7.5). Finally, our results can also inform observational campaigns that target outflows, indicating what properties of the outflows are connected to the presence or strength of certain species.

I thank the referee for an in-depth and constructive revision of the manuscript that has improved the presentation and analysis of the results. The initial inspiration for this work grew out of a stimulating discussion with Paul Martini during a visit supported by the Visitor Program at the Ohio State Center for Cosmology and Astroparticle Physics. I am grateful to him for valuable ideas and comments on this manuscript, and to the CCAP for kind hospitality. Thanks also to Renate Mauland for her work on the spectral stacks in this and our previous paper, and for several revisions of this manuscript. I am thankful to Ski Antonucci for enriching and valuable discussions on polarization in outflows, to Tzu-Ching Chang, Robert Wissing, Phil Berger, Sijing Shen, and Joop Schaye for conversations on shocks, waves and the outflow structure, and to Max Gronke for discussions on gas hydrodynamics. Thanks to Daniel Proga for a careful review of this manuscript and for providing many great comments, suggestions, and references, and to Stan Owocki for sharing his thoughts and notes on hydrostatic cloud modeling. I am grateful to Suoqing Ji, Fred Hamann, Sterl Phinney, Nick Scoville, Eliot Quataeret, Tom Barlow, and other colleagues at JPL and Caltech for many interesting comments and discussions. I am also thankful to the UCSB/MPIA ENIGMA group, for their kind hospitality and comments. This research was partially carried out at the Jet Propulsion Laboratory, California Institute of Technology, under a contract with the National Aeronautics and Space Administration.

Funding for SDSS-III has been provided by the Alfred P. Sloan Foundation, the Participating Institutions, the National Science Foundation, and the U.S. Department of Energy Office of Science. The SDSS-III website is http://www.sdss3.org/. SDSS-III is managed by the Astrophysical Research Consortium for the Participating Institutions of the SDSS-III Collaboration including the University of Arizona, the Brazilian Participation Group, Brookhaven National Laboratory, Carnegie Mellon University, University of Florida, the French Participation Group, the German Participation Group, Harvard University, the Instituto de Astrofisica de Canarias, the Michigan State/Notre Dame/JINA Participation Group, Johns Hopkins University, Lawrence Berkeley National Laboratory, Max Planck Institute for Astrophysics, Max Planck Institute for Extraterrestrial Physics, New Mexico State University, New York University, Ohio State University, Pennsylvania State University, University of Portsmouth, Princeton University, the Spanish Participation Group, University of Tokyo, University of Utah, Vanderbilt University, University of Virginia, University of Washington, and Yale University.

\section{Appendix \\ Equivalent Width Dependences}

This section presents the figures for the evolution of the equivalent widths with trough minimum velocity (Figure 9), quasar magnitude (Figure 10), and quasar redshift (Figure 11). 

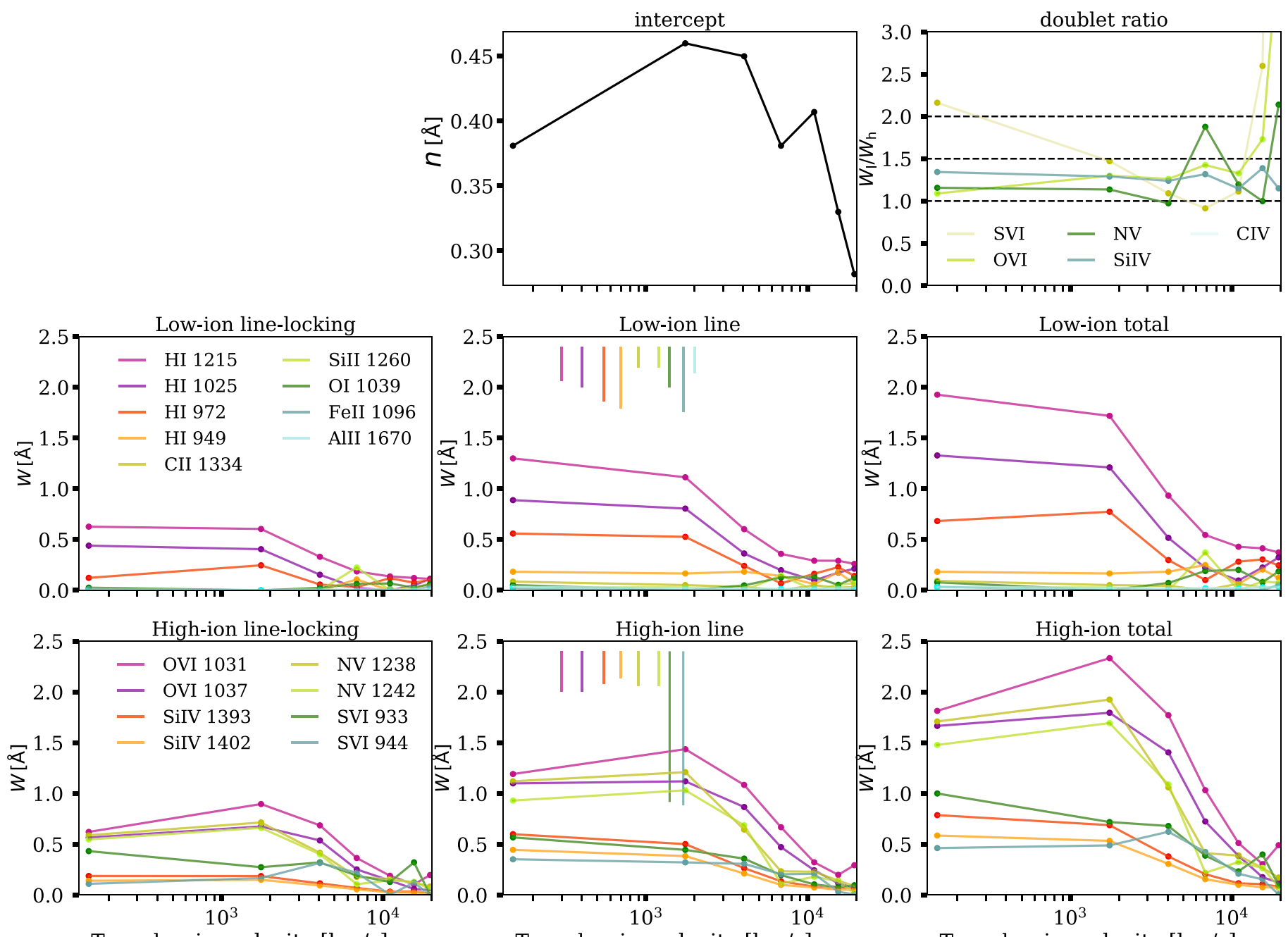

Trough $\min$. velocity $[\mathrm{km} / \mathrm{s}]$

Trough min. velocity $[\mathrm{km} / \mathrm{s}]$

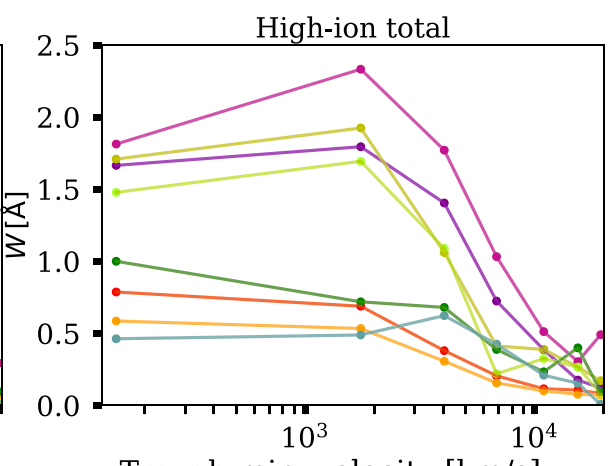

Trough min. velocity $[\mathrm{km} / \mathrm{s}]$

Figure 9. Evolution of the equivalent width as a function of trough minimum velocity, following the format of Figure 3 . There are no apparent differences beyond the uncertainty between troughs detached or not (smallest velocity bin) from the C IV emission line, other than the evolution already observed with outflow velocity. 

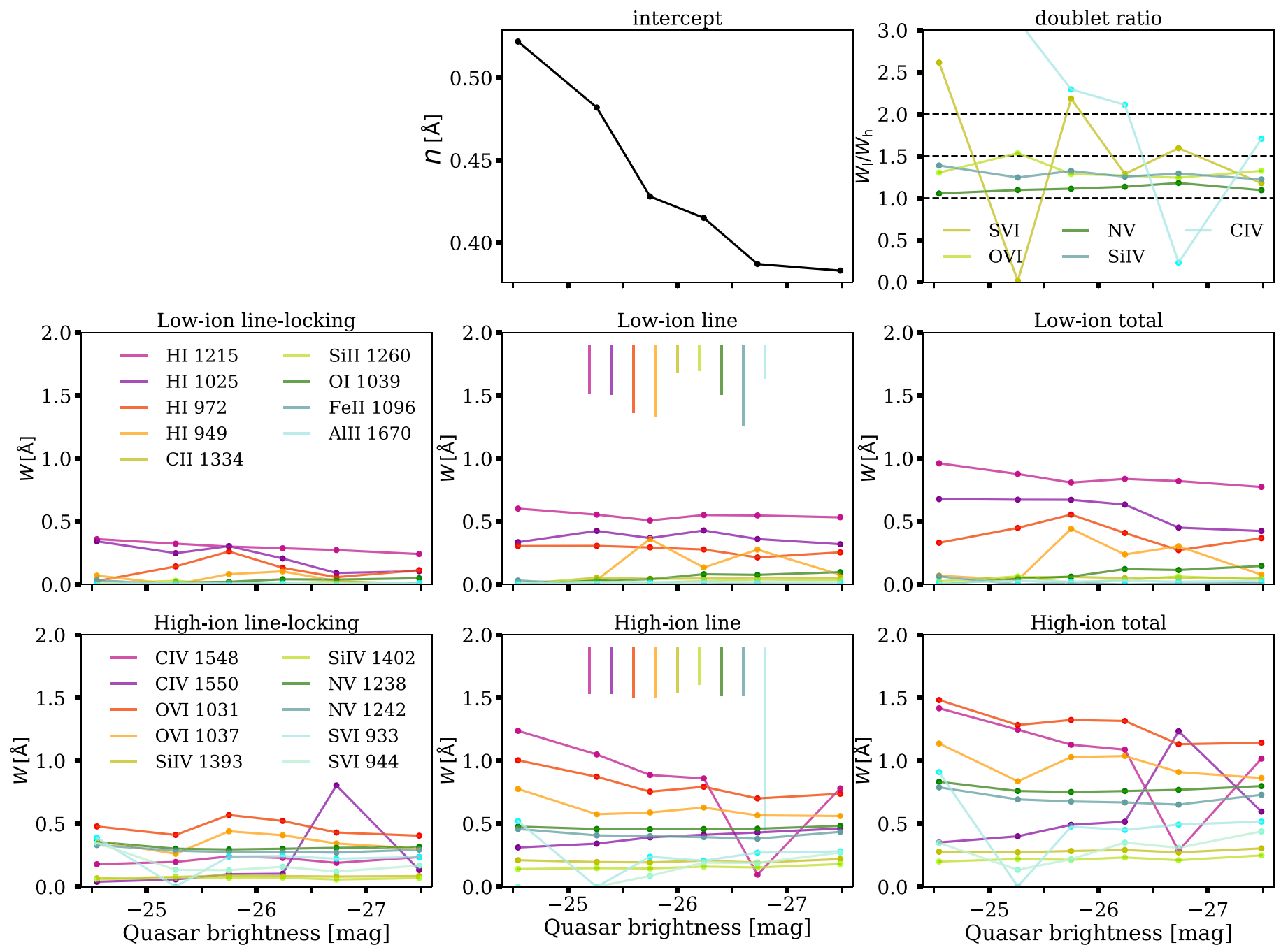

Figure 10. Evolution of the equivalent width as a function of quasar absolute magnitude, following the format of Figure 3 . There is no visible dependence of the equivalent width on the magnitude of the host quasar beyond the uncertainties. For some high-ionization species, a decrease of equivalent width with increasing brightness, consistent with the trend of the intercept, is suggested, albeit also within the uncertainty. 

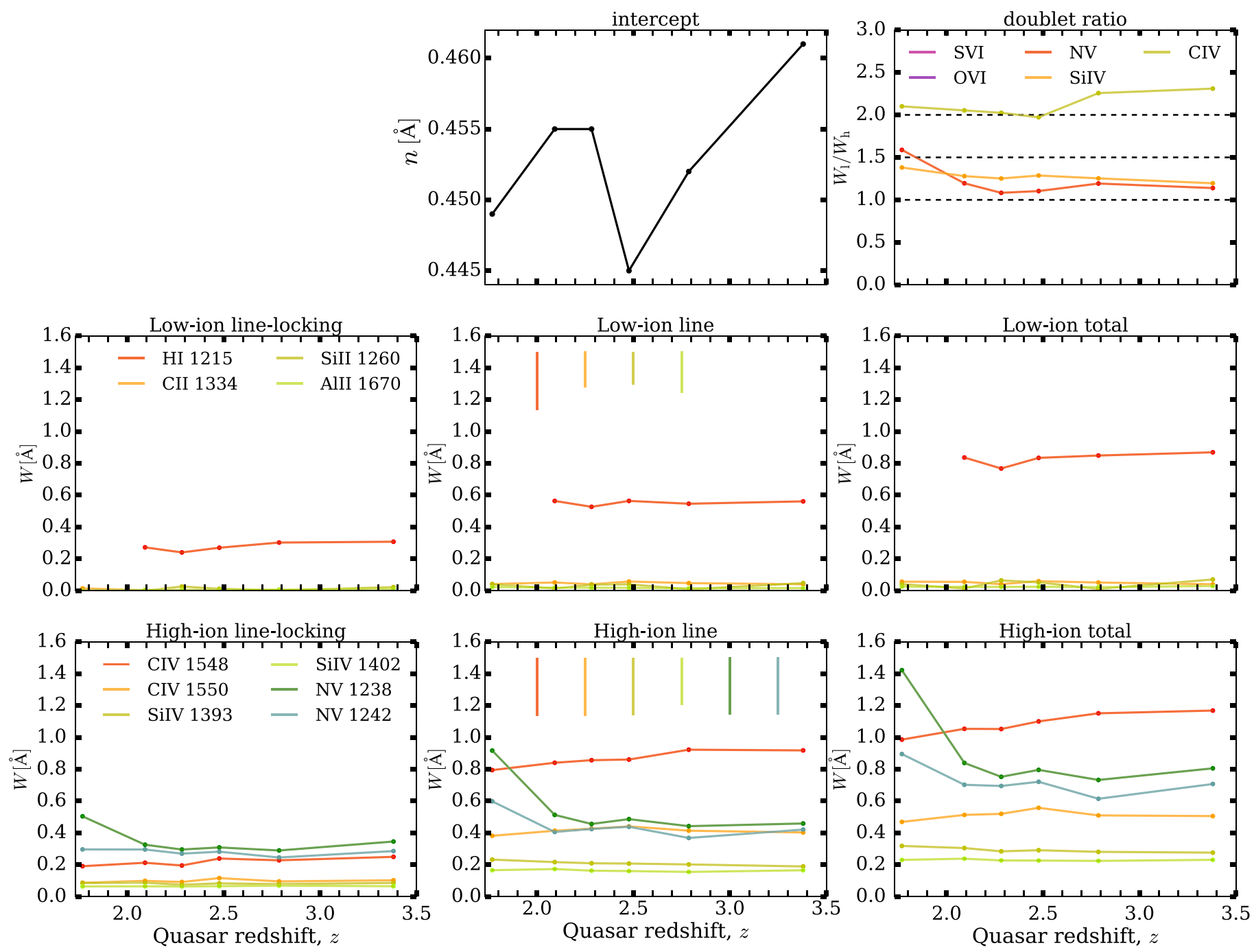

Figure 11. Evolution of the equivalent width as a function of redshift, following the format of Figure 3. In general, the measured equivalent widths remain constant with redshift, indicating that the local conditions of the radiation field and the physical state of the gas govern the observations.

\section{Spearman Rank Correlation Analysis}

Here, we quantify the correlation between the highionization species and the intercept " $n$ " for the evolution with outflow velocity (Figure 3 ). Figure 12 shows the results of a Spearman rank correlation analysis for these high ions. The values and uncertainties are the mean value and the standard deviation from the two lines of each doublet. The species C IV and Si IV present negative values of the correlation coefficient, which indicates an anticorrelation between their evolution and that of the intercept. A positive correlation is observed for O VI and $\mathrm{NV}$, as suggested in Section 6, although the correlation coefficient values are small: $\approx 0.2$ and $\approx 0.1$, respectively. The average value for $\mathrm{S} V I$ is also positive, $\approx 0.25$, but with large error bars consistent with no correlation $(\rho=0)$, due to the large differences in the evolution of the two lines of the doublet at small velocities (bottom panels in Figure 3). 


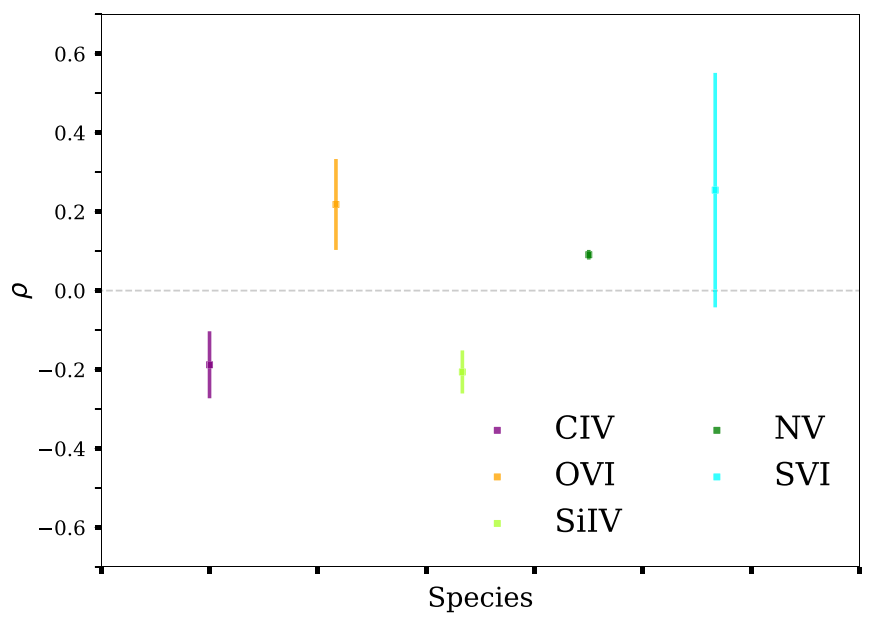

Figure 12. Values of the Spearman rank correlation analysis for the highionization species and the intercept with outflow velocity. Positive (negative) values of the correlation coefficient indicate correlation (anticorrelation) between the evolution of the high ions and the intercept, while a null value denotes no correlation. The species $\mathrm{O}$ VI, $\mathrm{N} \mathrm{V}$, and $\mathrm{S}$ VI present positive correlations, the latter with large error bars. See text for further details.

\section{ORCID iDs}

Lluís Mas-Ribas (iD https://orcid.org/0000-0003-4584-8841

\section{References}

Abbott, D. C. 1980, ApJ, 242, 1183

Allen, J. T., Hewett, P. C., Maddox, N., Richards, G. T., \& Belokurov, V. 2011, MNRAS, 410, 860

Arav, N., Becker, R. H., Laurent-Muehleisen, S. A., et al. 1999, ApJ, 524, 566

Arav, N., Borguet, B., Chamberlain, C., Edmonds, D., \& Danforth, C. 2013, MNRAS, 436, 3286

Arav, N., \& Li, Z.-Y. 1994, ApJ, 427, 700

Arav, N., Liu, G., Xu, X., et al. 2018, ApJ, 857, 60

Arinyo-i-Prats, A., Mas-Ribas, L., Miralda-Escudé, J., Pérez-Ràfols, I., \& Noterdaeme, P. 2018, MNRAS, 481, 3921

Baek, K. M., Bang, J. H., Jeon, Y.-K., Kang, S., \& Lee, H.-W. 2007, JKAS, 40,1

Baldwin, J. A. 1977, ApJ, 214, 679

Barnes, D. J., Kannan, R., Vogelsberger, M., \& Marinacci, F. 2018, arXiv: 1812.01611

Baskin, A., \& Laor, A. 2012, MNRAS, 426, 1144

Baskin, A., Laor, A., \& Hamann, F. 2013, MNRAS, 432, 1525

Baskin, A., Laor, A., \& Hamann, F. 2015, MNRAS, 449, 1593

Baskin, A., Laor, A., \& Stern, J. 2014a, MNRAS, 438, 604

Baskin, A., Laor, A., \& Stern, J. 2014b, MNRAS, 445, 3025

Bautista, J. E., Busca, N. G., Guy, J., et al. 2017, A\&A, 603, A12

Bonzini, M., Padovani, P., Mainieri, V., et al. 2017, MNRAS, 468, 4205

Borguet, B. C. J., Arav, N., Edmonds, D., Chamberlain, C., \& Benn, C. 2013, ApJ, 762, 49

Borguet, B. C. J., Edmonds, D., Arav, N., Benn, C., \& Chamberlain, C. 2012, ApJ, 758, 69

Bromage, G. E., Boksenberg, A., Clavel, J., et al. 1985, MNRAS, 215, 1

Busca, N. G., Delubac, T., Rich, J., et al. 2013, A\&A, 552, A96

Castor, J. I., Abbott, D. C., \& Klein, R. I. 1975, ApJ, 195, 157

Chamberlain, C., Arav, N., \& Benn, C. 2015, MNRAS, 450, 1085

Chang, S.-J., Heo, J.-E., Di Mille, F., et al. 2015, ApJ, 814, 98

Chang, S.-J., Lee, H.-W., \& Yang, Y. 2017, MNRAS, 464, 5018

Chartas, G., Brandt, W. N., \& Gallagher, S. C. 2003, ApJ, 595, 85

Chartas, G., Brandt, W. N., Gallagher, S. C., \& Garmire, G. P. 2002, ApJ, 579,169

Chartas, G., \& Canas, M. H. 2018, arXiv:1809.09138

Chelouche, D., \& Netzer, H. 2003, MNRAS, 344, 233

Choi, E., Ostriker, J. P., Naab, T., Oser, L., \& Moster, B. P. 2015, MNRAS, 449, 4105

Ciotti, L., Ostriker, J. P., \& Proga, D. 2010, ApJ, 717, 708

Dai, X., Shankar, F., \& Sivakoff, G. R. 2012, ApJ, 757, 180

Dannen, R. C., Proga, D., Kallman, T. R., \& Waters, T. 2019, ApJ, 882, 99
Davelaar, J., Bronzwaer, T., Kok, D., et al. 2018, arXiv:1811.08369 Dawson, K. S., Schlegel, D. J., Ahn, C. P., et al. 2013, AJ, 145, 10 de Kool, M. 1997, in ASP Conf. Ser. 128, Mass Ejection from Active Galactic Nuclei, ed. N. Arav, I. Shlosman, \& R. J. Weymann (San Francisco, CA: ASP), 233

de Kool, M., \& Begelman, M. C. 1995, ApJ, 455, 448

DESI Collaboration, Aghamousa, A., Aguilar, J., et al. 2016, arXiv:1611. 00036

DiPompeo, M. A., Brotherton, M. S., Becker, R. H., et al. 2010, ApJS, 189, 83

DiPompeo, M. A., Brotherton, M. S., \& De Breuck, C. 2013, MNRAS, 428, 1565

Dyda, S., Dannen, R., Waters, T., \& Proga, D. 2017, MNRAS, 467, 4161

Dyda, S., \& Proga, D. 2018, MNRAS, 478, 5006

Eisenstein, D. J., Weinberg, D. H., Agol, E., et al. 2011, AJ, 142, 72

Elvis, M. 2000, ApJ, 545, 63

Everett, J. E. 2005, ApJ, 631, 689

Faucher-Giguère, C.-A., Quataert, E., \& Murray, N. 2012, MNRAS, 420, 1347

Feldmeier, A., \& Shlosman, I. 2002, ApJ, 564, 385

Filiz Ak, N., Brandt, W. N., Hall, P. B., et al. 2013, ApJ, 777, 168

Filiz Ak, N., Brandt, W. N., Hall, P. B., et al. 2014, ApJ, 791, 88

Font-Ribera, A., Kirkby, D., Busca, N., et al. 2013, JCAP, 05, 027

Ganguly, R., \& Brotherton, M. S. 2008, ApJ, 672, 102

Ganguly, R., Brotherton, M. S., Cales, S., et al. 2007, ApJ, 665, 990

García-Burillo, S., Combes, F., Ramos Almeida, C., et al. 2016, ApJL, 823, L12

Gibson, R. R., Jiang, L., Brandt, W. N., et al. 2009, ApJ, 692, 758

Hall, P. B., Anderson, S. F., Strauss, M. A., et al. 2002, ApJS, 141, 267

Hamann, F. 1998, ApJ, 500, 798

Hamann, F., Chartas, G., McGraw, S., et al. 2013, MNRAS, 435, 133

Hamann, F., Chartas, G., Reeves, J., \& Nardini, E. 2018, MNRAS, 476, 943

Hamann, F., Herbst, H., Paris, I., \& Capellupo, D. 2019, MNRAS, 483, 1808

Hamann, F., Korista, K. T., \& Morris, S. L. 1993, ApJ, 415, 541

Hamann, F., \& Sabra, B. 2004, in ASP Conf. Ser. 311, AGN Physics with the Sloan Digital Sky Survey, ed. G. T. Richards \& P. B. Hall (San Francisco, CA: ASP), 203

Harrison, C. M., Costa, T., Tadhunter, C. N., et al. 2018, NatAs, 2, 198

He, Z., Wang, T., Zhou, H., et al. 2017, ApJS, 229, 22

Hewett, P. C., \& Foltz, C. B. 2003, AJ, 125, 1784

Higginbottom, N., Knigge, C., Long, K. S., Sim, S. A., \& Matthews, J. H. 2013, MNRAS, 436, 1390

Higginbottom, N., Proga, D., Knigge, C., et al. 2014, ApJ, 789, 19

Imanishi, M., Nakanishi, K., Izumi, T., \& Wada, K. 2018, ApJL, 853, L25

Izumi, T., Wada, K., Fukushige, R., Hamamura, S., \& Kohno, K. 2018, ApJ, 867,48

Kim, H. J., Lee, H.-W., \& Kang, S. 2007, MNRAS, 374, 187

King, A., \& Pounds, K. 2015, ARA\&A, 53, 115

Knigge, C., Scaringi, S., Goad, M. R., \& Cottis, C. E. 2008, MNRAS, 386,1426

Korista, K. T., Voit, G. M., Morris, S. L., \& Weymann, R. J. 1993, ApJS, 88,357

Kriss, G. A., Davidsen, A. F., Blair, W. P., et al. 1992, ApJ, 392, 485

Lamy, H., \& Hutsemékers, D. 2004, A\&A, 427, 107

Lee, H.-W., \& Blandford, R. D. 1997, MNRAS, 288, 19

Leighly, K. M. 2004, ApJ, 611, 125

Leighly, K. M., Hamann, F., Casebeer, D. A., \& Grupe, D. 2009, ApJ, 701, 176

Lynds, C. R. 1967, ApJ, 147, 396

Maksym, W. P., Fabbiano, G., Elvis, M., et al. 2019, ApJ, 872, 94

Mas-Ribas, L., \& Mauland, R. 2019, arXiv:1902.04085

Mas-Ribas, L., Miralda-Escudé, J., Pérez-Ràfols, I., et al. 2017, ApJ, 846, 4

Matthews, J. H., Knigge, C., Long, K. S., et al. 2016, MNRAS, 458, 293

McKernan, B., Yaqoob, T., \& Reynolds, C. S. 2007, MNRAS, 379, 1359

Milne, E. A. 1926, MNRAS, 86, 459

Misawa, T., Eracleous, M., Charlton, J. C., \& Kashikawa, N. 2018, arXiv: 1809.07476

Moravec, E. A., Hamann, F., Capellupo, D. M., et al. 2017, MNRAS, 468, 4539 Murray, N., Chiang, J., Grossman, S. A., \& Voit, G. M. 1995, ApJ, 451, 498

Nestor, D., Hamann, F., \& Rodriguez Hidalgo, P. 2008, MNRAS, 386, 2055

Ogle, P. M., Cohen, M. H., Miller, J. S., et al. 1999, ApJS, 125, 1

Pâris, I., Petitjean, P., Aubourg, É, et al. 2012, A\&A, 548, A66

Pâris, I., Petitjean, P., Ross, N. P., et al. 2017, A\&A, 597, A79

Parker, M. L., Alston, W. N., Buisson, D. J. K., et al. 2017, MNRAS, 469,1553

Pérez-Ràfols, I., Font-Ribera, A., Miralda-Escudé, J., et al. 2018a, MNRAS, 473, 3019

Pérez-Ràfols, I., Miralda-Escudé, J., Arinyo-i-Prats, A., Font-Ribera, A., \& Mas-Ribas, L. 2018b, MNRAS, 480, 4702 
Planck Collaboration, Ade, P. A. R., Aghanim, N., et al. 2016, A\&A, 594, A13 Proga, D., Dannen, R., Waters, T., \& Kallman, T. R. 2017, MNRAS, 467, 3160 Proga, D., \& Kallman, T. R. 2004, ApJ, 616, 688

Proga, D., Rodriguez-Hidalgo, P., \& Hamann, F. 2012, in ASP Conf. Ser. 460, AGN Winds in Charleston, ed. G. Chartas, F. Hamann, \& K. M. Leighly (San Francisco, CA: ASP), 171

Proga, D., Stone, J. M., \& Kallman, T. R. 2000, ApJ, 543, 686

Proga, D., \& Waters, T. 2015, ApJ, 804, 137

Reeves, J. N., Porquet, D., Braito, V., et al. 2013, ApJ, 776, 99

Reichard, T. A., Richards, G. T., Hall, P. B., et al. 2003, AJ, 126, 2594

Rodríguez Hidalgo, P., Hamann, F., \& Hall, P. 2011, MNRAS, 411, 247

Rogerson, J. A., Hall, P. B., Ahmed, N. S., et al. 2018, ApJ, 862, 22

Scargle, J. D. 1973, ApJ, 179, 705

Scoville, N., \& Norman, C. 1995, ApJ, 451, 510

Spilker, J. S., Aravena, M., Béthermin, M., et al. 2018, Sci, 361, 1016

Sprayberry, D., \& Foltz, C. B. 1992, ApJ, 390, 39

Stern, J., Behar, E., Laor, A., Baskin, A., \& Holczer, T. 2014, MNRAS, 445,3011
Stern, J., Hennawi, J. F., Prochaska, J. X., \& Werk, J. K. 2016, ApJ, 830, 87 Stevens, I. R., \& Kallman, T. R. 1990, ApJ, 365, 321

Sundqvist, J. O., Owocki, S. P., \& Puls, J. 2018, A\&A, 611, A17

Trump, J. R., Hall, P. B., Reichard, T. A., et al. 2006, ApJS, 165,

Tumlinson, J., Peeples, M. S., \& Werk, J. K. 2017, ARA\&A, 55, 389

Voit, G. M., Weymann, R. J., \& Korista, K. T. 1993, ApJ, 413, 95

Wang, H.-Y., Wang, T.-G., \& Wang, J.-X. 2005, ApJ, 634, 149

Wang, H.-Y., Wang, T.-G., \& Wang, J.-X. 2007, ApJS, 168, 195

Waters, T., \& Proga, D. 2016, MNRAS, 460, L79

Weinberger, R., Springel, V., Hernquist, L., et al. 2017, MNRAS, 465, 3291

Weymann, R. J., Carswell, R. F., \& Smith, M. G. 1981, ARA\&A, 19, 41

Weymann, R. J., Morris, S. L., Foltz, C. B., \& Hewett, P. C. 1991, ApJ, 373,23

Williamson, D., Venanzi, M., \& Hönig, S. 2018, arXiv:1812.07448

Wolfe, A. M., Gawiser, E., \& Prochaska, J. X. 2005, ARA\&A, 43, 861

Xu, X., Arav, N., Miller, T., \& Benn, C. 2018, ApJ, 858, 39

Xu, X., Arav, N., Miller, T., \& Benn, C. 2019, ApJ, 876, 105

York, D. G., Adelman, J., Anderson, J. E., Jr., et al. 2000, AJ, 120, 1579 